\title{
Statistical analyses of a screen cylinder wake
}

\author{
Azlin Mohd Azmi ${ }^{1,2}$, Tongming Zhou* ${ }^{1}$, Yu Zhou ${ }^{3}$, and Liang Cheng ${ }^{1}$ \\ ${ }^{1}$ School of Civil, Environmental and Mining Engineering, \\ The University of Western Australia, \\ 35 Stirling Highway, Crawley, WA 6009, Australia \\ 2 Faculty of Mechanical Engineering, Universiti Teknologi MARA, \\ Shah Alam, Selangor, Malaysia \\ ${ }^{3}$ Institute for Turbulence-Noise-Vibration Interaction and Control, Shen Zhen Graduate School, \\ Harbin Institute of Technology, P R China \\ *Corresponding Author: tongming.zhou@uwa.edu.au
}

\begin{abstract}
The evolution of a screen cylinder wake was studied by analysing its statistical properties over a streamwise range of $x / d=10-60$. The screen cylinder was made of a stainless steel screen mesh of $67 \%$ porosity. The experiments were conducted in a wind tunnel at a Reynolds number of 7000 using an X-probe. The results were compared with those obtained in the wake generated by a solid cylinder. It was observed that the evolution of the statistics in the wake of the screen cylinder was different from that of a solid cylinder, reflecting the differences in the formation of the organized large-scale vortices in both wakes. The streamwise evolution of the Reynolds stresses, energy spectra and cross-correlation coefficients indicated that there exists a critical location which differentiates the screen cylinder wake into two regions over the measured streamwise range. The formation of the fully-formed large-scale vortices was delayed until this critical location. Comparison with existing results for screen strips showed that although the near-wake characteristics and the vortex formation mechanism were similar between the two wake generators, variation of the Strouhal frequencies was observed and the self-preservation states were non-universal, reconfirming the dependence of a wake on its initial condition.
\end{abstract}


Keywords: Passive flow control; Vortex shedding suppression; Screen cylinder wake

\section{Introduction}

Flow through and around screens is significant in engineering applications where flow control is important, for example, for the purpose of suppressing flow-induced structural vibration, acoustic noise and hydrodynamic forces (Jaouen and Bécot, 2011, Klebert et al., 2013, Zdravkovich and Volk, 1972). A screen is a device used to manipulate fluid flow in which flow direction, time-averaged velocity non-uniformities and turbulence can be controlled. Common examples of screen are an array of parallel rods, honeycombs, perforated plates and wire-mesh screens. Various aspects of flow through screens can be found in the classical review of Laws and Livesey (1978).

The use of a screen mesh as a shroud for vortex shedding suppression was first explored by Zdravkovich and Volk (1972). They found that cylinders fitted with a fine-mesh gauze shroud were the most effective in suppressing VIV in comparison to the square-holed and circular-holed shrouds, all of $36 \%$ porosity, where the porosity is defined as the percentage of the open area over the total area. The gauze shroud affected the pressure distribution more significantly than the other two shrouds and the uniform part of the pressure distribution curve started at $120^{\circ}$ instead of $90^{\circ}$. The study on screen mesh as a controller of the bluff body wake has been revisited in recent years, e.g. Azmi et al. (2012), who found that the vibration amplitude of a bare cylinder was suppressed by about $50 \%$ by using a screen shroud of $72 \%$ porosity at a shroud-tocylinder diameter ratio of 1.25. Other investigations of screen mesh on vortex shedding suppression from a stationary cylinder were conducted in shallow water (e.g. Oruç, 2012, Ozkan et al., 2012, Ozkan et al., 2013). Oruç (2012) studied the wake of a circular cylinder surrounded 
by a streamlined thin-steel screen mesh of $50 \%$ porosity. It was found that significant vortex suppression could be achieved with the mesh. The turbulent statistics of the wake, such as turbulence intensity, Reynolds shear stress and turbulent kinetic energy, were drastically diminished in comparison to the bare cylinder wake. Ozkan et al. (2012) used a permeable cylinder made of a chrome-nickel wire mesh to examine the effect of porosity and diameter of the outer permeable cylinder on flow characteristics of the inner circular cylinder. They suggested that a better flow control was achieved with the outer-to-inner diameter ratio in the range of $1.6-2.0$ and mesh porosity in the range of $40-60 \%$. Ozkan et al. (2013) also showed that with a high screen porosity of $70 \%$, flow modification has been achieved where the vortex shedding frequency is considerably reduced as revealed by the spectra of the streamwise velocity fluctuations.

It is well-known that the porosity of a surface could modify the wake formation and reduces the intensity of vortex shedding. One of the earliest studies was conducted by Castro (1971) who found that by increasing the porosity of a plate, the recirculation bubble became detached from the plate and moved farther downstream. Two distinct wake flow regimes were identified: the Kármán vortex dominated wake at low porosity and the absence of Kármán vortices at porosity larger than 0.2. Alridge et al. (1978) examined a perforated circular cylinder of $60 \%$ porosity with variable aspect ratio $l / d$ (where $l$ is the cylinder length and $d$ is the cylinder diameter) at $R e$ (where $R e=U_{\infty} d / v$, with $U_{\infty}$ being the free stream velocity and $v$ being the kinematic viscosity of the fluid) between $10^{4}$ and $2.6 \times 10^{5}$. Flow visualisation conducted at $R e=3000$ in water showed the existence of small scale vortices and lack of correlation between the vortices in its wake. The studies on vortices behind a screen strip of $50 \%$ porosity indicated that the interaction 
of vortices appeared to be very weak and the coherent contribution to Reynolds shear stress is significant (Zhou and Antonia, 1992, 1995). Cannon et al. (1993) conducted flow visualizations to investigate the wakes of three circular screen disks with porosities of $50 \%, 40 \%$ and $15 \%$. It was found that the wakes of higher porosity screen disks were less irregular or contorted. Huang and Keffer (1996) reported that the formation region of the large-scale structures of their mesh strip of $40 \%$ porosity can be extended to $20 h$ downstream, where $h$ is the height of the strip. Extension of the work in the far-wake region showed that the spanwise coherent structures and the lateral coherent structures were indeed connected, suggesting that the far-wake structures were evolved directly from the near-wake structures, rather than from the wake instability (Huang et al., 1996). In a recent study, Takeuchi et al. (2007) measured the airflows around permeable cylinders made of wire mesh with porosities of $0.54 \%$ and $0.71 \%$ rolled in circle. The flow just behind the permeable cylinders (> 50\% porosity) had forward velocity due to the permeability, which alleviated the near field excitation of Kármán vortex shedding. As a result, the reattachment point behind the cylinder was delayed several times of that of the solid cylinder wake. Gansel et al. (2011) tested cylinders made from metal mesh of various porosities. The results, in contrast to Takeuchi et al. (2007), indicated that the wake characteristics changed toward the wake characteristics of a solid cylinder at a porosity just below $75 \%$. Levy et al. (2014) argued that the surface porosity was not the key parameter in defining the flow topology of a porous screen, rather, a non-dimensional parameter based on the twine thickness, mesh hole size and cylinder diameter might influence their experimental data.

Several numerical investigations were also reported on the wake of porous body. Bhattacharyya et al. (2006) studied the fluid flow and concentration field in and around a permeable cylinder 
and found that the flow field remained steady for the range of low Reynolds number considered. The drag experienced by the porous cylinder reduced monotonically with the increase of Reynolds number and the decrease of Darcy number $D a\left(\equiv K / d^{2}\right.$, where $K$ is the permeability of the medium). The two parameters, namely the porosity and permeability, are closely related. In general, higher porosity in a material is likely to be accompanied by higher permeability also. Whereas porosity is a measure of how much open space exists within a solid, permeability is the measure of the ease with which a fluid can pass through the porous body. The vorticity contours at $D a=10^{-2}$ showed weak vortex strength in comparison with that of a solid cylinder wake. Analyses on the flow structures around square and circular permeable cylinders were also conducted for a range of Reynolds numbers, Darcy numbers and porosities (Yu et al., 2010, 2011). The results showed that the recirculating wake existing downstream of the cylinders was completely detached from the bodies under a certain range of parameters.

The various studies on porous and perforated bodies proved the low strength vortices shed from these bodies. However, very limited studies have been conducted so far in understanding the formation and development of a screen cylinder wake. Antonia et al. (2002) speculated that it was unlikely for a screen cylinder to exhibit the same flow topology as a screen strip although the autocorrelation coefficients of the velocity signals showed some strong quasi-periodicity in both the screen strip and the screen cylinder wakes. Analysis on a screen cylinder wake would certainly complement the relatively limited literature on this topic. The present study aims to gain initial insight into the development of a screen cylinder wake by examining the streamwise evolution of the statistics and to compare the results with those reported previously. However, due to the lack of reported results on porous cylinders, comparisons of the results will be made 
only with those obtained in the wakes of screen strips. More detailed investigations on the formation and development of the large-scale coherent structures in the screen cylinder wake using phase-averaged analysis can be found in (Azmi, 2015).

\section{Experimental Details}

The experiments were conducted in an open circuit wind tunnel with a test section of $380 \mathrm{~mm}$ (width) $\times 255 \mathrm{~mm}$ (height) and $1.8 \mathrm{~m}$ (long). All interior surfaces of the test section were made of smooth acrylic to minimise the boundary layer effects. The free stream velocity in the test section was uniform to $0.2 \%$, and the longitudinal turbulence intensity was less than $0.5 \%$. Two types of cylinders were used for the purpose of this study, i.e. a solid cylinder and a screen cylinder. The diameter $d$ of the solid cylinder was $10.1 \mathrm{~mm}$ while that of the screen cylinder was $21 \mathrm{~mm}$. All measurements were performed at $R e=7000$, corresponding to a free stream velocity $U_{\infty}$ of $10.4 \mathrm{~m} / \mathrm{s}$ and $5 \mathrm{~m} / \mathrm{s}$ for the solid cylinder and the screen cylinder, respectively. Both cylinders were located $15 \mathrm{~cm}$ downstream of the entrance of the test section and installed in the mid-plane and spanned the whole width of the wind tunnel. Plastic plugs were inserted and flush with the tunnel inner wall to prevent leakage at the ends. Previous studies (e.g. Kubo et al., 1989; Fox and West, 1990; Szepessy and Bearman, 1992) have shown the importance of end plates in obtaining two-dimensional vortex shedding. Therefore, in the present study, two end plates were also used for both cylinders. Following the suggestions by Stansby (1974), the dimension of the end plates was $7 d \times 7 d$. Fig. 1 shows the wake profile and the arrangement of the screen cylinder. The coordinate system is defined such that the $x$-axis is in the same direction of the incoming flow, $y$-axis is in the vertical plane through the mid-span of the cylinder and normal to the $x$-axis and $z$-axis is normal to both the $x$ and $y$-axes. The cylinders were fixed $2.5 d$ from the 
leading edge of the end plates. The thickness of the plates was $1.25 \mathrm{~mm}$ with the leading and trailing edges being sharpened at an angle of $45^{\circ}$. They were fixed on the tunnel side walls by using four pieces of acrylic blocks on each side. The size of the acrylic blocks was $4 \mathrm{~mm}$ (height) $\times 3 \mathrm{~mm}$ (width) $\times 20 \mathrm{~mm}$ (length) to keep the end plates about $4 \mathrm{~mm}$ away from the tunnel walls. Such a distance warrants the end plates to be located above the boundary layer. The length $l$ of both cylinders between the end plates was about $370 \mathrm{~mm}$, giving aspect ratios $l / d$ of 37 for the solid cylinder and 17.6 for the screen cylinder. The solid cylinder was a plain and smooth cylinder made of polished aluminium. The screen cylinder was made of a stainless steel wire mesh of aperture $a=2 \mathrm{~mm}$ and wire diameter $d_{w}=0.45 \mathrm{~mm}$. The porosity of the screen mesh, $\beta$, which is defined as the ratio of the opening area to the total area, i.e. $a^{2} /\left(a+d_{w}\right)^{2}$, was $67 \%$. Prior to making the screen cylinder, a length of $66 \mathrm{~mm}$ (i.e. $\pi d$ ) was marked on a screen mesh, which was then enfolded into a cylindrical form making sure that the mesh had an appropriate curvature even at the pre-cutting edge. The screen cylinder was then cut through the middle of the aperture along the wire (i.e. along the cylinder axial direction, no wire was cut across except those aligned perpendicular to the axial direction). The two edges of the screen cylinder were then tied off using a $0.16 \mathrm{~mm}$ diameter wire every $30-40 \mathrm{~mm}$ along the cylinder axial direction. Due to the small diameter of the wire, the ties are too small to affect the flow apparently. Care was also taken to make sure that its diameter was uniform along the cylinder axis (with a tolerance less than $\pm 1 \%$ ). It was monitored during the tests that no apparent deformation was observed. Preliminary experiments conducted with the joint line orientated at $0^{\circ}$ and $180^{\circ}$ from the cylinder trailing edge gave similar results in the mean and root-mean-square values of the velocities (with a scatter less than $\pm 1 \%$ ), making the effect of the joint orientation to be negligible. 
The measurement locations were at $x^{*}=10-60$, where a superscript asterisk denotes normalisation by $U_{\infty}$ and/or $d$. For the purpose of examining the streamwise evolution of the wakes, an X-probe was used to measure the longitudinal and transverse velocity fluctuations $u$ and $v$, respectively. In order to examine the evolution of the correlation length of the large-scale structures in the spanwise direction, two X-probes located at $y^{*}=0.5$ were used with one probe being fixed at $11 \mathrm{~cm}$ from the left side wall of the tunnel and the other moved along the cylinder axial direction. The separation between the probes was in the range of $5 \mathrm{~mm}$ and $155 \mathrm{~mm}$. For these experiments, the measurement locations were $x^{*}=5-60$.

The hot wires were etched to an active length of $1 \mathrm{~mm}$ from Wollaston (Pt-10\% Rh) wires. Each of the two wires in the X-probe had a diameter of $5 \mu \mathrm{m}$. The X-probe was calibrated using the effective angle method. Details of this method can be found in Bradshaw (1971) and Browne et al. (1988). The angle calibration was performed over $\pm 20^{\circ}$. The effective angles of the inclined wires in the X-probe were $34^{\circ}$ and $31^{\circ}$, respectively. The hot wires were operated with in-house constant temperature circuits at an over heat ratio of 1.5. The output signals were low-pass filtered at a cut-off frequency $f_{c}$ set at $5.2 \mathrm{kHz}$. The filtered signals were sampled at a frequency $f_{s}=2 f_{c}$ into a $\mathrm{PC}$ using a 16 -bit $\mathrm{A} / \mathrm{D}$ converter. The sampling period $T_{s}$ was $30 \mathrm{~s}$. Experimental uncertainties were inferred from the hot-wire calibration data errors as well as the scatter observed in repeating the experiments a number of times. The uncertainty for the timeaverage velocity, $\bar{U}$, was estimated to be about $\pm 2 \%$ while for the root-mean-square values of the fluctuation velocities $u$ and $v$ and the Reynolds shear stress $\langle u v\rangle$, the uncertainties were about $\pm 5 \%, \pm 6 \%$ and $\pm 8 \%$, respectively, where angular brackets denote time-averaging. 
Smoke wire flow visualizations in the same wind tunnel were also carried out using a high speed camera to obtain a qualitative understanding on the evolution of vortices in the wake of the screen cylinder. The visualizations were conducted at Reynolds numbers of 1240, 1920 and 7000. However, due to quick smoke dispersion in the wake at $R e=7000$ (e.g. Lee et al., 2004) and hence the low quality of the images, only images at $R e=1240$ and 1920 will be shown in the present paper.

\section{Results and discussion}

\subsection{Mean and root-mean-square (rms) velocities}

The maximum velocity defect is defined as $U_{o}=U_{\infty}-U_{\min }$ and the wake half-width $L$ is defined as the distance from the wake centerline to the point where the velocity defect is $U_{o} / 2$ (Fig. 1). Table 1 shows the values of $U_{o}$ and $L$ for both cylinder wakes, normalised by $U_{\infty}$ and $d$ as denoted by a superscript asterisk. Results from Antonia and Mi (1998) for wakes of a solid cylinder and a screen strip (50\% porosity) at $R e=3000$ are also included in the table. These results would be helpful in understanding the general flow pattern of the screen bodies although the Reynolds number and the porosity are slightly lower in their study than those in the present one. The study by Antonia and Mi (1998) suggested that the screen strip wake (50\% porosity) approached self-preservation much more rapidly than the solid cylinder wake. The screen cylinder wake is studied in a similar manner to that of Antonia and Mi (1998). The normalized velocity defects $\left(U_{\infty}-\bar{U}\right) / U_{o}$ for the wakes of the solid cylinder and the screen cylinder are shown in Fig. 2. The mean velocity defect distribution obtained in the far-wake ( $x^{*}=420$ at $R e$ = 1170) of a circular cylinder by Browne et al. (1987) is also included. There is reasonable agreement of the data at different streamwise locations, especially in the region of $y / L<1.2$. 
However, the agreement of the distributions $\left(U_{\infty}-\bar{U}\right) / U_{o}$ in different wakes at the centre region is forced due to the normalisation. As pointed out by Antonia and Mi (1998), it is difficult to infer whether self-preservation has been attained in the flows, especially in the near wake region, due to the existence of the large-scale structures in the solid cylinder wake. As a result, we can say that the mean velocity defect is at least not a sensitive indicator of self-preservation. A more sensitive indicator of self-preservation may be provided by the Reynolds stresses.

Results of $u^{\prime} / U_{o}, v^{\prime} / U_{o}$ and $\left\langle u v>/ U_{o}{ }^{2}\right.$ at different downstream locations are shown in Figs. 3,4 and 5, respectively, where a prime indicates the root-mean-square (rms) values. The results of Browne et al. (1987) obtained in the far-wake of a solid cylinder are also shown in these figures. For the solid cylinder wake, $u^{\prime} / U_{o}$ decreases with $x^{*}$, indicating a decaying trend of the turbulence intensity in the solid cylinder wake, consistent with the trend of Antonia and $\mathrm{Mi}$ (1998). To avoid crowding the figures, results from Antonia and Mi (1998) are not included. Fig. 3(a) also shows that the self-preservation state has not been reached in the solid cylinder wake. This is similar to that of Antonia and Mi (1998), who argued that the asymptotic state was likely to be reached at several hundred diameters downstream as their rms values of the velocity components were still developing at each location, which were significantly larger than the distribution of Browne et al. (1987). For the screen cylinder wake (Fig. 3b), the trend is in contrast to that of a solid cylinder, i.e., $u^{\prime} / U_{o}$ increases with $x^{*}$, at least up to $x^{*}=50$, indicating enhancement of the turbulent intensity and expansion of the wake region as evolving downstream. Considering the experimental uncertainty of $u^{\prime}$, which is about $\pm 5 \%$, it seems that self-preservation has been attained in the context of $u^{\prime} / U_{o}$ at $x^{*} \approx 50$, where $u^{\prime} / U_{o}$ collapse beyond this location, although they are below the distribution of Browne et al. (1987). In 
comparing with the screen strip wake, the distributions of $u^{\prime} / U_{o}$ of Antonia and Mi (1998) overlap at $x^{*} \geq 60$. The values of $u^{\prime} / U_{o}$ beyond this location only show reasonable agreement with that of Browne et al. (1987) in the central part of the wake.

For the distributions of $v^{\prime} / U_{o}$ in the screen cylinder wake (Fig. 4b), similar to $u^{\prime} / U_{o}$, they increase with $x^{*}$, which is in contrast to that of the solid cylinder wake (Fig. 4a). The agreement of $v^{\prime} / U_{o}$ for $x^{*} \geq 50$ is very satisfactory, indicating that self-preservation might be attained in the context of $v^{\prime} / U_{o}$. The magnitudes of $v^{\prime} / U_{o}$ in the present study for $x^{*} \geq 50$ are just slightly larger than that obtained in the screen strip wake after self-preservation is attained (Antonia \& Mi 1998) and both distributions are above the far-wake distribution of Browne et al. (1987). This result indicates that even though self-preservation state is achieved in different wakes, this state does not necessarily have a universal expression. It should also reflect the influence of the initial conditions or the type of wake generators.

The normalized Reynolds shear stress $<u v>/ U_{o}{ }^{2}$ (Fig. 5a) for the solid cylinder wake decreases with the increase of streamwise locations, indicating that self-preservation is far to be achieved. For the screen cylinder wake (Fig. 5b), however, it increases as evolving downstream till about at $x^{*}=50$, indicating that self-preservation is likely to be attained at this location, which supports the observations in Figs. 3(b) and 4(b). In addition, the values of $\langle u v\rangle / U_{o}{ }^{2}$ in the self-preserving region of the screen cylinder wake is about $12 \%$ smaller than that in the solid cylinder far-wake (Browne et al., 1987) and 25\% higher than that in the screen strip wake (Antonia \& Mi, 1998), supporting again the non-universality of the self-preservation state for different wake generators. 
Before examining other statistical results, it is pertinent to discuss the streamwise variation of $U_{0}$ and $L$. The present maximum velocity defect and the wake half-width for the solid cylinder wake are comparable with those reported by Antonia and Mi (1998) (Table 1). The maximum velocity defect in the screen cylinder wake is significantly larger than that in the solid cylinder wake (see also Fig. 6a) and decreases quickly with $x^{*}$. Interestingly, the decreasing trend of $U_{0}^{*}$ in the screen cylinder wake is similar to that in the screen strip wake of Antonia and Mi (1998). The distributions of $L^{*}$ show good agreement in both screen wakes. They are about half of that of the solid cylinder wake at the measured locations. The distributions of $L^{*}$ increase linearly with $x^{*}$ in all the wakes (Fig. 6b), for the measured streamwise range. However, the linear region for the screen strip wake and the screen cylinder wake appear to start at $x^{*}=20$. The slope of $L^{*}$ in the solid cylinder wake $(0.028)$ is much higher than that in the screen cylinder wake $(0.0154)$ and the screen strip wake $(0.012)$, indicating that the wake region of the solid cylinder expands much faster than that of the other two wakes. It needs to be noted that a power law relationship $L \sim x^{0.5}$ is expected in the far wake when self-preservation is achieved (Sreenivasan, 1981). However, due to the limited streamwise range covered in the current experiments, curve fitting to $L$ in the self-preservation region is not available.

Fig. 7 presents the streamwise evolution of the rms values $u^{\prime}$ and $v^{\prime}$ normalized by the velocity deficit on the centreline of the wakes to show the self-preservation behaviour. The results from Browne et al. (1987) in the far-wake region after self-preservation is achieved are also included in the figure. The values of $u^{\prime} / U_{o}$ and $v^{\prime} / U_{o}$ decrease non-linearly with $x^{*}$ in the solid cylinder wakes but increase in the wakes of screen cylinder and screen strip. Interestingly, the values of 
$u^{\prime} / U_{o}$ and $v^{\prime} / U_{o}$ for $x^{*}<40$ fall on different sides of the far-wake self-preservation counterparts, one above (solid cylinder wake) and the other below (screen cylinder and screen strip wakes). This observation might imply the same mechanism for the development in the Reynolds normal stresses of the two screen wakes. This is also indicative of structural differences between the respective studied wakes. While the Kármán vortices in the solid cylinder wake are decaying, the vortices in the screen cylinder (and also the screen strip) wake are developing up to $x^{*}=40$ based on the results of $u^{\prime} / U_{o}$ and $v^{\prime} / U_{o}$. After this location, vortices in the screen cylinder wake are fully developed. The above results also highlight the differences in the approach to self-preservation in the solid cylinder wake and the two screen wakes, in which the latter achieve self-preservation much more rapidly than the former due to the weaker large eddies (Sreenivasan, 1981). The higher values of $u^{\prime} / U_{o}$ and $v^{\prime} / U_{o}$ in the screen cylinder wakes $\left(x^{*} \geq 40\right)$ than that in the solid cylinder far-wake (Browne et al., 1987) suggest the non-universality of self-preserving state for different wake generators.

\subsection{Velocity signals and energy spectra}

Figs. 8(a,b) show the velocity signals for the transverse velocity component $v$ obtained at $y^{*}=0.5$ in the solid cylinder and the screen cylinder wakes at $x^{*}=10$. Apparent periodicity is evident in the solid cylinder wake (Fig. 8a), implying a noticeable periodic shedding of vortices whereas for the screen cylinder wake (Fig. 8b), the signal exhibits strong fluctuation at high frequency. In addition to the figures above, the signal in the screen cylinder wake at $x^{*}=40$ (Fig. 8c) is also shown to highlight the differences of the $v$ signals at different streamwise locations. The periodicity with low frequency is evident at $x^{*}=40$, implying the shedding of organized-vortices although with much smaller strength in comparison to the strong Kármán 
Vortex Street in the solid cylinder wake.

The vortex shedding frequency $f$ can be determined from the energy spectral density function $\phi_{v}$ obtained by conducting Fast Fourier Transform (FFT) to the velocity signals. It is defined such that $\int_{0}^{\infty} \phi_{v}(x) d x=1$, where $x$ represents frequency. The frequency resolution of the energy spectra depends on the sampling frequency and the window size when performing FFT. Using a window size of $2^{13}$, the frequency resolution of the power spectra is about $0.63 \mathrm{~Hz}$. The spectra $\phi_{v}$ for both wakes obtained at $y^{*}=0.5$ for various streamwise locations are shown in Fig. 9. This is in accordance with the previous study of Zhou et al. (2003) who showed that the vortex centre of a solid cylinder wake for $x^{*}=10$ was located at $y^{*}=0.5$. The spectra obtained along the outer edge of the screen cylinder wake are also displayed in Fig. 9(c). Each spectrum has been shifted downward by one order relative to the one above it for easy viewing. For the solid cylinder wake (Fig. 9a), Kármán vortex shedding at $x^{*}=10$ is apparent at a frequency $f$ of 201 $\mathrm{Hz}$, corresponding to $f^{*}\left(\equiv f d / U_{\infty}\right)=0.2$, consistent with existing literatures of a solid cylinder wake at subcritical Reynolds numbers. At farther downstream locations, e.g. $x^{*}=20$, the peak height reduces although $f$ remains the same, and only a very minor peak is visible at $x^{*}=40$. This trend indicates that Kármán vortices are decaying as evolving downstream. On the other hand, the energy spectra of the screen cylinder wake (Fig. 9b) reveal no apparent peak at $x^{*}=10$, suggesting that there are no organised structures at these locations, which highlights the difference in the near-wake behaviour of the two wakes. A minor broad-peak is only visible at $x^{*}=20$ occurring at a frequency $f^{*} \approx 0.277$, indicating the occurrence of some periodicity of the organised structures. The nominal frequencies associated with the peak in the screen cylinder 
wake represent the characteristic frequencies of the vortices in the shear layers. The peak at $f^{*}=$ 0.257 becomes most apparent at $x^{*}=30$, indicating the formation of the large-scale structures. For $x^{*}=30 \sim 60$, the peak frequency decreases from 0.257 to 0.234 . On the other hand, the spectra along the outer edge (Fig. 9c) display broad-band peaks even in the near wake, indicating the existence of periodicity at all measured downstream locations. Huang and Keffer (1996) reported the same behaviour for their screen strip wake $\left(\beta=40 \%\right.$ and $\left.R e=1.1 \times 10^{4}\right)$ at $x^{*}=1$ and attributed this to the Kelvin-Helmholtz instability of vortex sheets generated at the mesh edge and the subsequent rolled up of the sheets into discrete vortices, similar to the single shear layer development. The periodicity becomes more apparent and the peak magnitude becomes larger from $x^{*}=10-30$. The decrease of the broad peak location towards lower frequencies along the streamwise direction indicates the growth of the vortical structures within the shear layers. It is hypothesized that this behaviour is due to an increase in the average wavelength, assuming slowly increasing convective velocity. The above process may imply merging of these vortices, which accounts for the formation of the large-scale structures in the screen cylinder wake, similar to the findings of Huang and Keffer (1996) and Antonia and Mi (1998). The continuous decrease of the peak frequency indicates that the merging process does not occur at a fixed downstream location. The delay in the formation of the large-scale structures is also similar to the available literatures on the wake of a porous body (Cimbala et al., 1988, Wygnanski et al., 1986). Huang and Keffer (1996) found that the formation region of the largescale structures in their screen strip wake can be extended to $x^{*}=20$. They reported that at $x^{*}=24$, the predominate broad-band peak on the energy spectra resulting from the quasiperiodic occurrence of the large-scale structures occurred at $f^{*}=0.188$, while in the present experiment for the screen cylinder wake, the predominant peak occurs at $x^{*}=40$ with $f^{*}=$ 
0.26. The differences in the formation length and the vortex shedding frequency of both the screen strip and the screen cylinder wakes obviously related to their underlying differences in mesh geometry, porosity and/or Reynolds number.

The streamwise variations of the peak frequency in various wakes are shown in Fig. 10. The Strouhal frequency in the solid cylinder wake is constant $(0.2)$ over $x^{*}=10 \rightarrow 40$. Previous studies (Zhou et al., 2003) on a solid cylinder wake have shown that the Kármán vortices decay nearly completely at $x^{*}=40$. However, in the screen cylinder wake, continuous frequency decay can be observed as evolving downstream. It is believed that the apparent decrease of the peak frequency in the screen cylinder wake is due to the merging of the small-scale vortices in the shear layers to form the large ones (which will also be discussed later based on flow visualisation result). After the formation of the large-scale structures, they evolve downstream at a slightly decreased frequency. The results of the screen strip wake inferred from Antonia and $\mathrm{Mi}$ (1998) and the screen strip wake of Huang and Keffer (1996) are also included in Fig. 10. Both results show that the peak frequency continues to shift to lower values as $x^{*}$ increases. It seems that the decrease of the peak frequency in the screen cylinder and the screen strip wakes can be classified into two regimes. In the first regime, a quick decrease in peak frequency implies a merging process of the small-scale vortices to form relatively larger ones. In the second regime, the frequency decrease is much slower than that in the first one. This gradual decrease should be related with the decay of the organised structures due to viscous effect.

\subsection{Autocorrelation Coefficients}

The autocorrelation coefficients $\rho_{\alpha}$ can also be used to examine the periodicity and highlight the 
differences of the organized structures in both the solid cylinder and the screen cylinder wakes. It is defined as:

$$
\rho_{\alpha}=\langle\alpha(x) \alpha(x+r)\rangle /\left\langle\alpha^{2}\right\rangle
$$

where $\alpha$ represents any velocity component and $r$ is the longitudinal separation between two points which can be calculated based on Taylor's hypothesis by converting a time delay $\tau$ to a streamwise separation through $r=\tau U_{\infty}$. The autocorrelation coefficients $\rho_{v}$ obtained in both solid cylinder and screen cylinder wakes are shown in Fig. 11. In the solid cylinder wake, $\rho_{v}$ shows more apparent periodicity with a much larger magnitude than that in the screen cylinder wake, especially at $x^{*}=10$. The regular oscillation of $\rho_{v}$ indicates a well-defined Kármán vortex street in the near-wake region. At further downstream locations, $\rho_{v}$ decreases significantly. At about $x^{*}=40$, the oscillation of $\rho_{v}$ is very small, indicating the complete decay of the Kármán vortex street. By contrast, at this location, $\rho_{v}$ in the screen cylinder wake approaches zero

quickly at about $r^{*}=2$ in comparison with that of the solid cylinder, indicating the absence of the large coherent structures immediately downstream of the screen cylinder. The periodicity starts to become noticeable at $x^{*}=20$, but with a much less magnitude than that in the solid cylinder wake and approaches to zero at about $r^{*}=10$ in comparison to that in the solid cylinder wake where marked periodicity still continues over a long distance. For $x^{*} \geq 30$, the periodicity of $\rho_{v}$ in the screen cylinder wake is more apparent with large magnitude and the distributions collapse really well with each other.

\subsection{Cross-correlation coefficients}

The cross-correlation coefficients between two velocity signals separated by a distance $\Delta \mathrm{z}$ can be used to quantify the three-dimensional flow characteristics of the vortical structures in the 
spanwise direction. It is defined as:

$$
\rho_{\alpha_{1}, \alpha_{2}}=\max \left\{\frac{\left|\left\langle\alpha_{1}(z, t) \cdot \alpha_{2}(z+\Delta z, t+\tau)\right\rangle\right|}{\sigma_{v_{1}} \sigma_{v_{2}}}\right\}
$$

where the time delay $\tau$ lies on the domain $[-0.5 / f, 0.5 / f], f$ is the vortex shedding frequency, $\sigma_{v_{1}}$ and $\sigma_{v_{2}}$ are the standard deviations of $v_{1}$ and $v_{2}$, respectively, and $\Delta \mathrm{z}$ is the separation between the two velocity signals. The cross-correlation coefficients between $v_{1}$ and $v_{2}$ at various downstream locations are shown in Fig. 12. The distributions of $\rho_{v_{1}, v_{2}}$ for both the solid cylinder and the screen cylinder wakes illustrate a decreasing correlation with the increase of probe separation. Moreover, this decrease appears to be the larger the farther downstream of the measurement locations in the wake of the solid cylinder due to the breakdown of the large-scale vortical structures. After $x^{*} \geq 40$, the decrease of $\rho_{v_{1}, v_{2}}$ is not apparent. Also included in Fig. 12(a) are the results for a solid cylinder wake from Zhou et al. (2011), which were obtained using hot wire probes (located at $x^{*}=5$ ) at $R e=20,430$. The agreement between the two studies is satisfactory for $\Delta \mathrm{z}^{*} \leq 1.5$. At larger probe separations, the values in Zhou et al. (2011) are much smaller than the present results. This could be due to the much higher Reynolds number and freestream turbulent intensity (about 2\%) in the former study, resulting in a faster vortex breakdown process. In contrast to a decreasing trend in the solid cylinder wake, the screen cylinder wake behaves in quite the opposite manner - the correlation coefficient increases with the increase of the downstream locations. For example, at $x^{*}=5$ and 10 , there is nearly no correlation between the two velocity signals, which is consistent with the finding that there are no large-scale vortical structures detected in the near wake at $y^{*}=0.5$. There seems a sudden increase in the correlation coefficient at $x^{*}=20$ and 30, which indicates that at these locations, the small-scale vortices in the shear layers may have started to merge together to form the largescale organised structures. This result is consistent with that shown in Figs. 9(b) and 9(c). The 
different trends of $\rho_{v_{1}, v_{2}}$ in the two wakes are indicative of decreasing size of the vortex structures in the solid cylinder wake and increasing size in the screen cylinder wake when evolving downstream. At downstream locations of $x^{*} \geq 40$, the cross-correlation coefficient remains unchanged, which likely shows the existence of the large-scale structures in this region.

Previous studies have shown that vortices shed from a cylinder are in cells in the turbulent regime. The average length of the cells can be quantified using the so-called correlation length. There are a few definitions for it. The most common one is that defined by Norberg (2003) as $\Lambda=\int_{0}^{\infty} \rho_{\alpha_{1}, \alpha_{2}} d z$. There are also some other definitions about the correlation length, such as the one by $\mathrm{Xu}$ et al. (2008) who defined it as the separation when the cross-correlation coefficient is equal to 0.5 . In the present paper, the correlation length is calculated using Norberg's definition and the results are shown in Fig. 13. It can be seen that $\Lambda$ in the solid cylinder wake decreases quickly, especially between $x^{*}=10$ and 20 , decreasing from $3.6 d$ to $1.8 d$. For $\Lambda$ in the screen cylinder wake, it is roughly a small constant value for $x^{*}=5-10$, which is in agreement with the previous discussions and the visualisation result (which will be shown in the next section) that there exist only small-scale eddies in the shear layers. The correlation length increases quickly between $x^{*}=10$ and 40 , indicating the merging process of the small-scale structures to form the larger-scale ones in the wake. For $x^{*} \geq 40, \Lambda$ is approximately a constant value with a variation of only about $\pm 3 \%$. The gradient of $\Lambda$ in the streamwise direction for the screen cylinder wake, $d \Lambda / d x$, is also shown in Fig. 13 on the right ordinate. It can be seen that the maximum rate of increase of $\Lambda$ occurs at around $x^{*}=23$, which may indicate the transition from the small-scale shear layer vortices to the large-scale vortex street. In addition, even at $x^{*} \geq 40$, the correlation length in the screen cylinder wake is smaller than that in the solid cylinder wake 
at $x^{*}=10$ and 20, indicating a more three-dimensionality nature of the flow in the former wake than that in the latter.

\subsection{Flow visualization in both cylinder wakes}

To confirm the above results related with spectra and cross-correlation coefficients, flow visualisation using smoke wire technique in a wind tunnel are also conducted at Reynolds numbers of about 1240 and 1920 as shown in Fig. 14. Such low Reynolds numbers are chosen for flow visualization in order to obtain better quality images. These results are only expected to qualitatively illustrate the vortex development at the respective $R e$ and thus must be used with caution. The flow pattern may be indicative to the one at $R e=7000$, but the vortex formation length may be different as will be discussed later. For the solid cylinder wake (Fig. 14a), the large-scale organized vortices can be seen clearly with two rows of counter-rotating vortex structures connected by the streamwise rib-like structures. The rib-like structures are stretched due to the counter-rotating vortices. The two rows of vortices also interact with each other when evolve downstream by penetrating to the opposite side across the wake centreline. The formation length of the Kármán vortices is about $2 d$, which is in agreement with that reported by Unal and Rockwell (1988). The screen cylinder wake clearly shows two shear layers in the upper and lower wake region, where small-scale vortices are formed at about $8 d$ (Fig. 14b). These vortex structures are much smaller in size and higher in frequency than the Kármán vortices in the solid cylinder wake. They do not roll up quickly to form the large-scale structures. As evolving downstream, the small-scale structures become larger in size, indicating a change in the wavelength characteristics. It is conjectured that when their sizes are large enough, they engulf the one next to them, resulting in amalgamation. At the same time, the onset of the interaction 
between the two shear layers can be seen at about $x^{*}=13$. At $x^{*}=20$, the smoke disappears due to dispersion and the large-scale structures are completely formed (Fig. 14c). Another flow visualization conducted at $R e=1920$ (Fig. 14d) shows small-scale structures slightly farther downstream $\left(x^{*}=10\right)$ compared with that at $R e=1240$. The interaction between the shear layers is delayed, indicating that the formation length becomes larger as $R e$ increases. Cimbala et al. (1988) reported a similar flow pattern for their screen strip wake of 53\% porosity at $R e=$ 6000. They noticed a fairly regular vortex street pattern at $x^{*} \approx 30$ in the screen strip wake. Obviously these types of flows show similar pattern of the growth of vortices from small-scale to the large-scale ones, with the difference being in the formation length/region. The latter is expected to depend on factors such as the geometry of the porous/perforated body, porosity and Reynolds number. From the visualization results, it can be concluded that the formation length becomes larger as $R e$ increases. Nevertheless, the above visualization supports the statistical analyses which show the possibility of vortex merging process and the delay of the large-scale organized structures in the screen cylinder wake.

\section{Conclusions}

Statistical characteristics in the wake of a screen cylinder made of a stainless steel mesh of $67 \%$ porosity are investigated in a wind tunnel using an X-probe over a region of $x^{*}=10-60$. The main aim of this study is to gain some general insight about the wake formation and evolution in the streamwise direction. The results are also compared with those of solid cylinder and screen strip wakes. The main conclusions are summarized as follows:

(1) The rms values $u^{\prime}$ and $v^{\prime}$ on the centreline of the screen cylinder wake increase linearly towards the self-preserving state. While the Kármán vortices in the solid cylinder wake 
are decaying, the vortices in the screen cylinder wake are growing up to a critical location $\left(x^{*}=40\right)$, beyond which the vortices are fully formed.

(2) The peak frequency on the energy spectra for the screen cylinder wake shifts to lower values with increasing downstream locations, similar to that in a screen strip wake. This phenomenon may be attributed to the amalgamation of the small-scale vortices and the formation of the larger-scale vortices farther downstream although there are differences in vortex shedding frequency observed in all the screen body wakes, which is expected due to the difference in porosity, screen geometry and Reynolds number.

(3) The autocorrelation coefficients of $v$ show no periodic characteristics in the screen cylinder wake for $x^{*} \leq 10$. After this location, there is some quasi-periodicity which subsides over time in contrast to the apparent periodicity exhibited by the solid cylinder wake that persists over a long distance.

(4) For the screen cylinder wake, the increase of the cross-correlation coefficients between two velocity signals $v_{1}$ and $v_{2}$ and the spanwise correlation length with downstream distance indicates the transition from the small-scale shear layer vortices to the largescale vortices. The maximum increasing rate of the spanwise correlation length occurs at about $x^{*}=23$, which may mark the initial interaction between the shear layers at this location.

(5) The mechanism of vortex formation in a screen cylinder wake is similar to that in a screen strip wake, which possibly involves the vortex merging process although the flow details downstream of the formation region may probably be different. The studied screen cylinder made of $67 \%$ porosity wire mesh is effective in subduing and delaying the formation of the large-scale vortex structures in its wake. 


\section{Acknowledgements}

AMA wishes to acknowledge financial assistance from UWA completion scholarship, the Ministry of Higher Education Malaysia (Fundamental Research Grant Scheme) and Universiti Teknologi MARA (Lestari). The authors sincerely acknowledge the financial supports from Australian Research Council through Projects DP110105171 and DP130104535.

\section{References}

Alridge, T.R., Piper, B.S., Hunt, J.C.R., 1978. The drag coefficient of finite-aspect-ratio perforated circular cylinders. J. Wind Eng Ind Aerod. 3: 251-57.

Antonia, R.A., Mi, J., 1998. Approach towards self-preservation of turbulent cylinder and screen wakes. Exp. Therm Fluid Sci. 17: 277-84.

Antonia, R.A., Zhou, T., Romano, G.P., 2002. Small-scale turbulence characteristics of twodimensional bluff body wakes. J. Fluid Mech. 459: 67-92.

Azmi, A.M., 2015. Wake characteristics of a screen cylinder and a screen shrouded cylinder. Doctor of Philosophy University of Western Australia.

Azmi, A.M., Zhou, T., Wang, H., Chua, L. P., Cheng, L., On the effectiveness and mechanism of vortex induced vibration suppression using a screen cylinder. Twenty-second International Offshore and Polar Engineering Conference, Rhodes Greece, June 17-22, 
2012.

Bhattacharyya, S., Dhinakaran, S., Khalili, A., 2006. Fluid motion around and through a porous cylinder. Chem. Eng. Sci. 61: 4451-61.

Bradshaw, P., 1971. An introduction to turbulence and its measurement Oxford Pergamon Press.

Browne, L.W.B., Antonia, R.A., Chua, L.P., 1989. Calibration ofx-probes for turbulent flow measurements. Exp. Fluids 7: 201-08.

Browne, L.W.B., Antonia, R.A., Shah, D.A., 1987. Turbulent energy-dissipation in a wake. J. Fluid Mech. 179: 307-26.

Cannon, S., Champagne, F., Glezer, A., 1993. Observations of large-scale structures in wakes behind axisymmetric bodies. Exp. Fluids 14: 447-50.

Castro, I.P., 1971. Wake characteristics of two dimensional perforated plates normal to an airstream. J. Fluid Mech. 46: 599-609.

Cimbala, J.M., Nagib, H.M., Roshko, A., 1988. Large structure in the far wakes of twodimensional bluff bodies. J. Fluid Mech. 190: 265-98.

Fox, T.A., West, G.S., 1990. On the use of end plates with circular cylinders. Exp. Fluids 9: 23739.

Gansel, L.C., McClimans, T.A., Myrhaug, D., 2011. The effects of fish cages on ambient currents. J. Offshore Mech Arct. 134: 011303-011303.

Huang, Z., Kawall, J.G., Keffer, J.F., 1996. Development of structure within the turbulent wake of a porous body. Part 2. Evolution of the three-dimensional features. J. Fluid Mech. 329: 117-36.

Huang, Z., Keffer, J.F., 1996. Development of structure within the turbulent wake of a porous body. Part 1. The initial formation region. J. Fluid Mech. 329: 103-15. 
Jaouen, L., Bécot, F.X., 2011. Acoustical characterization of perforated facings. J. Acoust. Soc. Am. 129: 1400-06.

Klebert, P., Lader, P., Gansel, L., Oppedal, F., 2013. Hydrodynamic interactions on net panel and aquaculture fish cages: A review. Ocean Eng. 58: 260-74.

Kubo, Y., Miyazaki, M., Kato, K., 1989. Effects of end plates and blockage of structural members on drag forces. J. Wind Eng Ind Aerod. 32: 329-42.

Laws, E.M., Livesey, J.L., 1978. Flow through screens. Annu. Rev. Fluid Mech. 10: 247-66.

Lee, S.J., Lee, S.I, Park, C.W., 2004. Reducinh the drag on a circular cylinder by upstream installation of a small control rod. Fluid Dyn. Res. 34, 233-250.

Levy, B., Friedrich, H., Cater, J.E., Clarke, R.J., Denier, J.P., 2014. The impact of twine/mesh ratio on the flow dynamics through a porous cylinder. Exp. Fluids 55: 1-11.

Norberg, C., 2003. Fluctuating lift on a circular cylinder: Review and new measurements. J. Fluid Struct. 17: 57-96.

Oruç, V., 2012. Passive control of flow structures around a circular cylinder by using screen. J. Fluid Struct. 33: 229-42.

Ozkan, G.M., Akilli, H., Sahin, B., 2013. Effect of high porosity screen on the near wake of a circular cylinder. EPJ Web of Conferences 45: 01071-p.1-01071-p.5

Ozkan, G., Oruç, V., Akilli, H., Sahin, B., 2012. Flow around a cylinder surrounded by a permeable cylinder in shallow water. Exp. Fluids 53: 1751-63.

Stansby, P.K., 1974. The effects of end plates on the base pressure coefficient of a circular cylinder. R. Aeronaut. J 78: 36-37.

Sreenivasan, K.R., 1981. Approach to self-preservation in plane turbulent wakes. AIAA J. 19: 1365-67. 
Szepessy, S., Bearman, P.W., 1992. Aspect ratio and end plate effects on vortex shedding from a circular cylinder. J. Fluid Mech. 234: 191-217.

Takeuchi, H., Tasaka, Y., Murai, Y., Takeda, Y., Tezuka, H., Mori, M., Particle image velocimetry for air flows behind permeable cylinders. ASME/JSME 5th Joint Fluids Engineering Conference, San Diego, California, July 30-August 2, 2007.

Unal, M.F., Rockwell, D., 1988. On vortex formation from a cylinder. Part 1. The initial instability. J. Fluid Mech. 190: 491-512.

Wygnanski, I., Champagne, F., Marasli, B., 1986. On the large-scale structures in twodimensional, small-deficit, turbulent wakes. J. Fluid Mech. 168: 31-71.

Xu, S.J., Zhou, Y., Tu, J.Y., 2008. Two-dimensionality of a cantilevered-cylinder wake in the presence of an oscillating upstream cylinder. J. Fluid Struc. 24: 467-80.

Yu, P., Zeng, Y., Lee, T.S., Bai, H.X., Low, H.T., 2010. Wake structure for flow past and through a porous square cylinder. Int. J. Heat Fluid Flow 31: 141-53.

Yu, P., Zeng, Y., Lee, T.S., Chen, X.B., Low, H.T., 2011. Steady flow around and through a permeable circular cylinder. Computers \& Fluids 42: 1-12.

Zdravkovich, M.M., Volk, J.R., 1972. Effect of shroud geometry on pressure distributed around a circular cylinder. J. Sound Vib. 20: 451-55.

Zhou, Y., Antonia, R.A., 1992. Topology and transport characteristics of vortices in a turbulent wake - effect of initial conditions. $11^{\text {th }}$ Australasian Fluid Mechanics Conference, Hobart Australia, Dec 14-18, 1992.

Zhou, Y., Antonia, R.A., 1995. Memory effects in a turbulent plane wake Exp. Fluids 19: 11220.

Zhou, T., Razali, S.F.M., Hao, Z., Cheng, L., 2011. On the study of vortex-induced vibration of a 
cylinder with helical strakes. J. Fluid Struct. 27: 903-17.

Zhou, T., Zhou, Y., Yiu, M.W., Chua, L.P., 2003. Three-dimensional vorticity in a turbulent cylinder wake. Exp. Fluids 35: 459-71.

Table 1. Maximum velocity defect and wake half-width

\begin{tabular}{|c|c|c|c|c|c|c|c|c|}
\hline \multicolumn{5}{|c|}{$\begin{array}{l}\text { Velocity defect, } \\
U_{o}{ }^{*}=U_{o} / U_{\infty}\end{array}$} & \multicolumn{4}{|c|}{$\begin{array}{l}\text { Wake half-width, } \\
\qquad L^{*}=L / d\end{array}$} \\
\hline$x^{*}$ & $\begin{array}{l}\text { Solid } \\
\text { cylinder } \\
\text { (Antonia } \\
\text { and Mi, } \\
\text { 1998) }\end{array}$ & $\begin{array}{l}\text { Present } \\
\text { solid } \\
\text { cylinder }\end{array}$ & $\begin{array}{l}\text { Screen } \\
\text { strip } \\
\text { (Antonia } \\
\text { and Mi, } \\
\text { 1998) }\end{array}$ & $\begin{array}{l}\text { Present } \\
\text { screen } \\
\text { cylinder }\end{array}$ & $\begin{array}{l}\text { Solid } \\
\text { cylinder } \\
\text { (Antonia } \\
\text { and Mi, } \\
\text { 1998) }\end{array}$ & $\begin{array}{l}\text { Present } \\
\text { solid } \\
\text { cylinder }\end{array}$ & $\begin{array}{l}\text { Screen strip } \\
\text { (Antonia and } \\
\text { Mi, 1998) }\end{array}$ & $\begin{array}{l}\text { Present } \\
\text { screen } \\
\text { cylinder }\end{array}$ \\
\hline 10 & 0.22 & 0.21 & 0.56 & 0.49 & 0.68 & 0.77 & 0.41 & 0.51 \\
\hline 20 & 0.19 & 0.19 & 0.30 & 0.43 & 0.87 & 0.99 & 0.63 & 0.52 \\
\hline 30 & & 0.18 & & 0.32 & & 1.28 & & 0.65 \\
\hline 40 & 0.16 & 0.15 & 0.16 & 0.23 & 1.50 & 1.56 & 0.88 & 0.82 \\
\hline 50 & & 0.14 & & 0.18 & & 1.92 & & 0.95 \\
\hline 60 & & 0.12 & 0.13 & 0.16 & & 2.09 & 1.08 & 1.07 \\
\hline
\end{tabular}


Figures

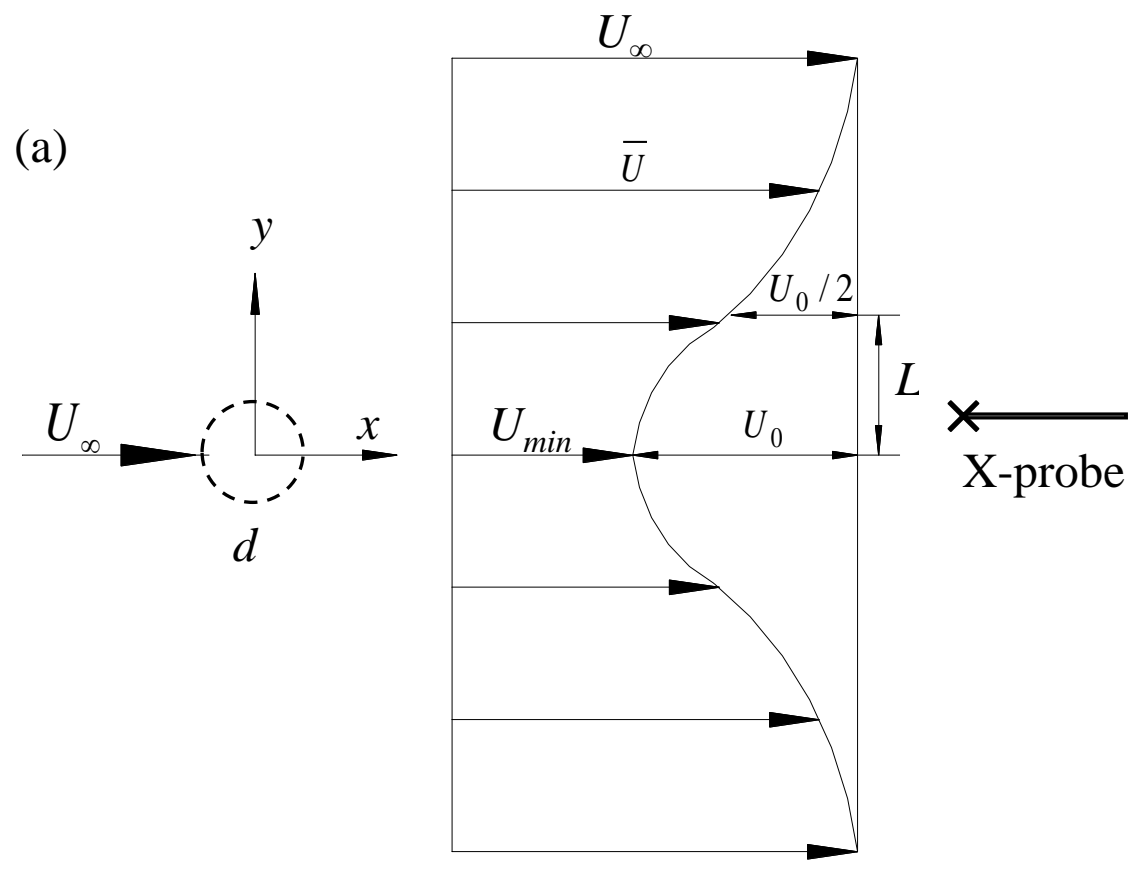




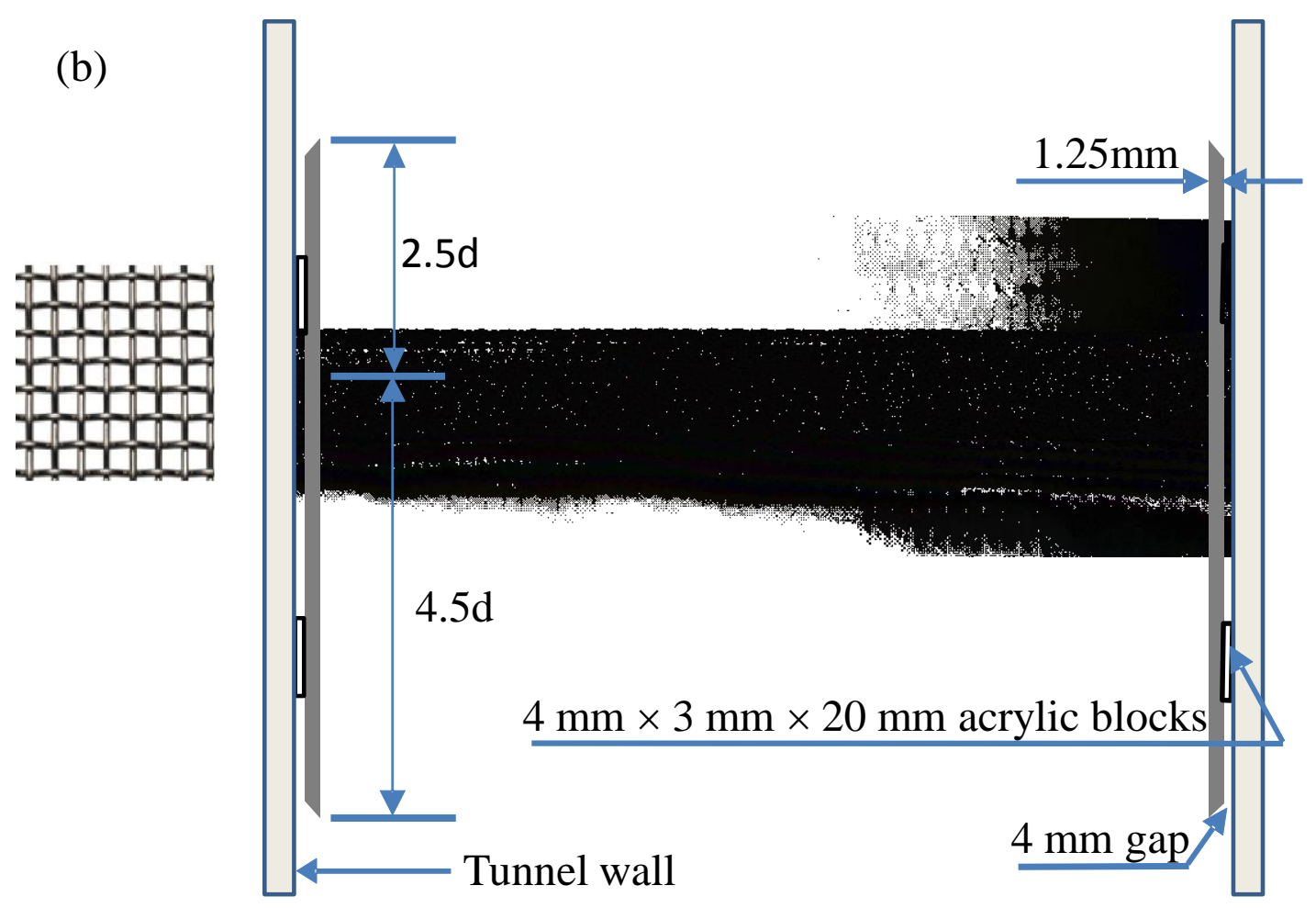

Fig. 1. Co-ordinate system, wake profile and probe arrangement (a) and the top view of the arrangement of the screen cylinder (b). Please note that the figures are not drawn to scale. 

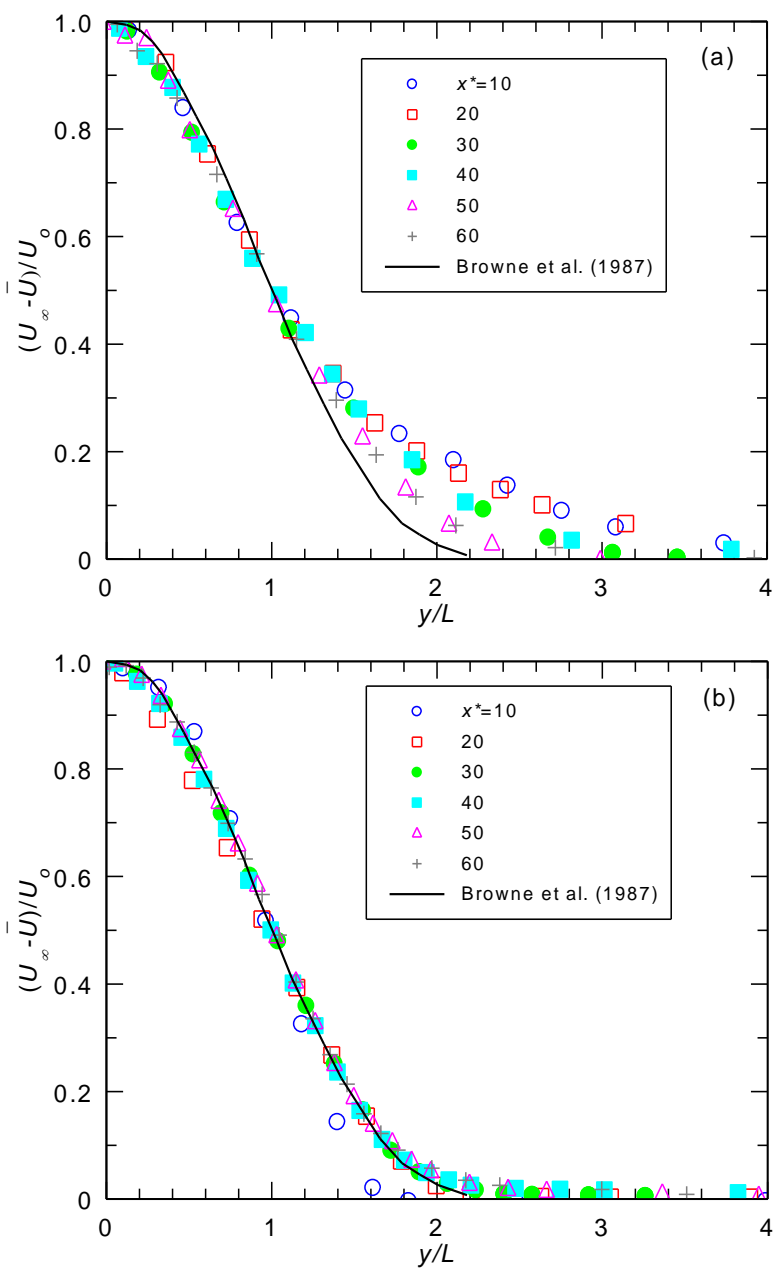

Fig. 2. Streamwise evolution of the normalized mean velocity defect. (a) solid cylinder; (b) screen cylinder. The solid lines represent values of the solid cylinder far wake at $x^{*}=420$ where self-preservation is achieved (Browne et al., 1987).

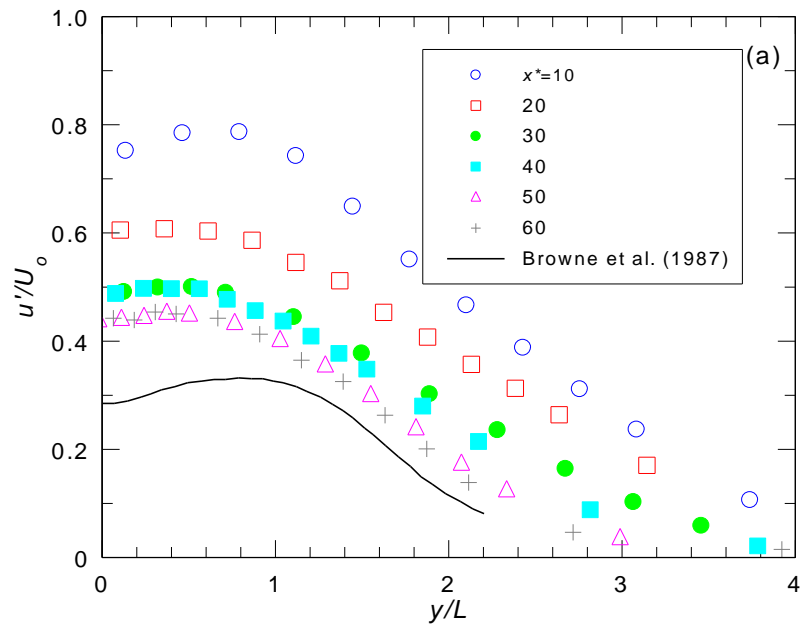




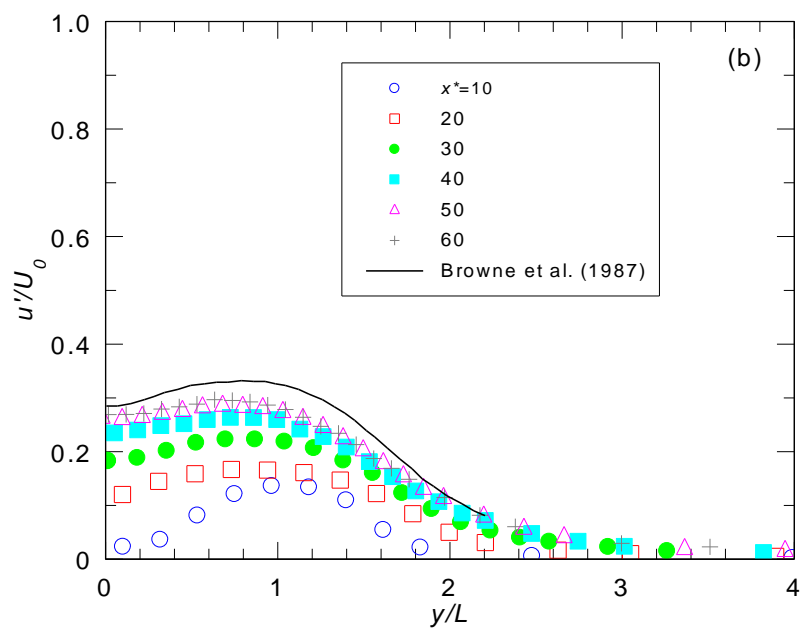

Fig. 3. Streamwise evolution of the normalized rms longitudinal velocity. (a) solid cylinder; (b) screen cylinder. The solid lines represent values of the solid cylinder far wake at $x^{*}=420$ where self-preservation is achieved (Browne et al., 1987).
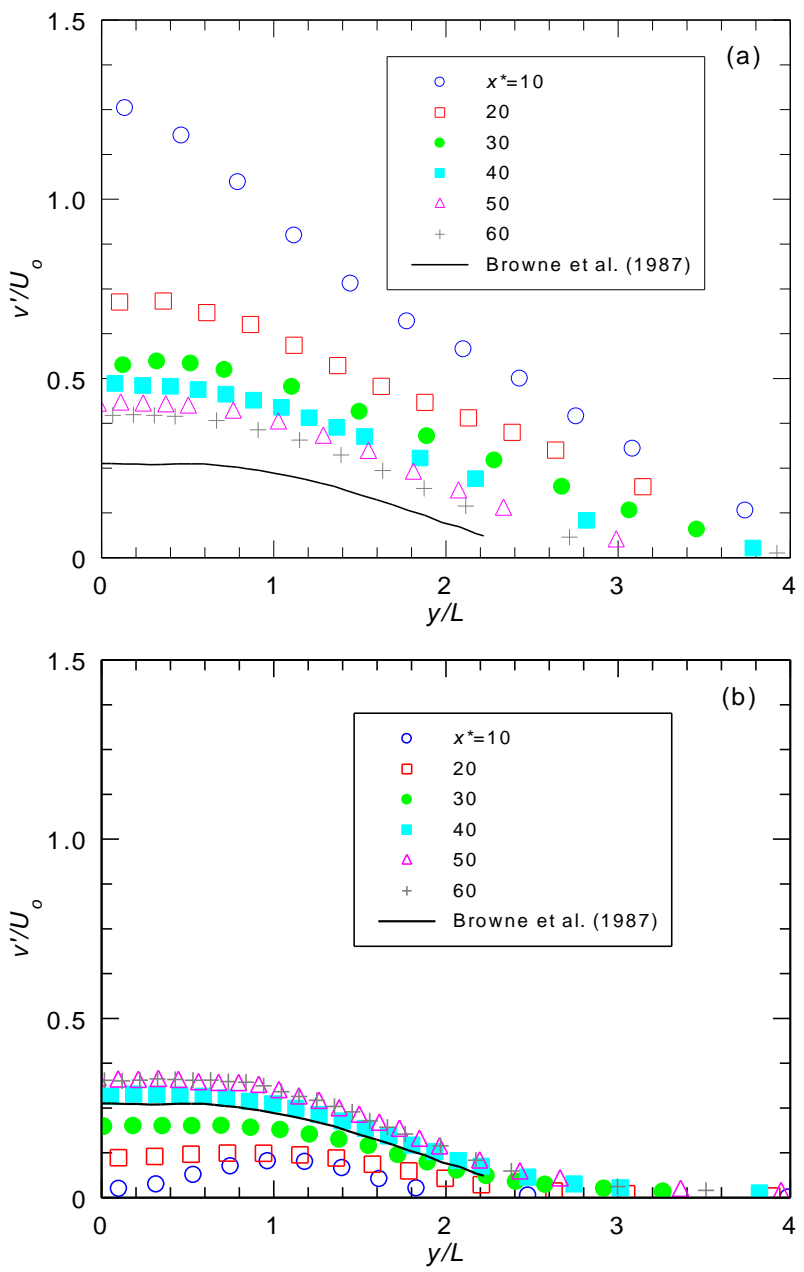

Fig. 4. Streamwise evolution of the normalized rms lateral velocity. (a) solid cylinder; (b) screen cylinder. The solid lines represent values of the solid cylinder far wake at $x^{*}=420$ where self-preservation is achieved (Browne et al., 1987). 

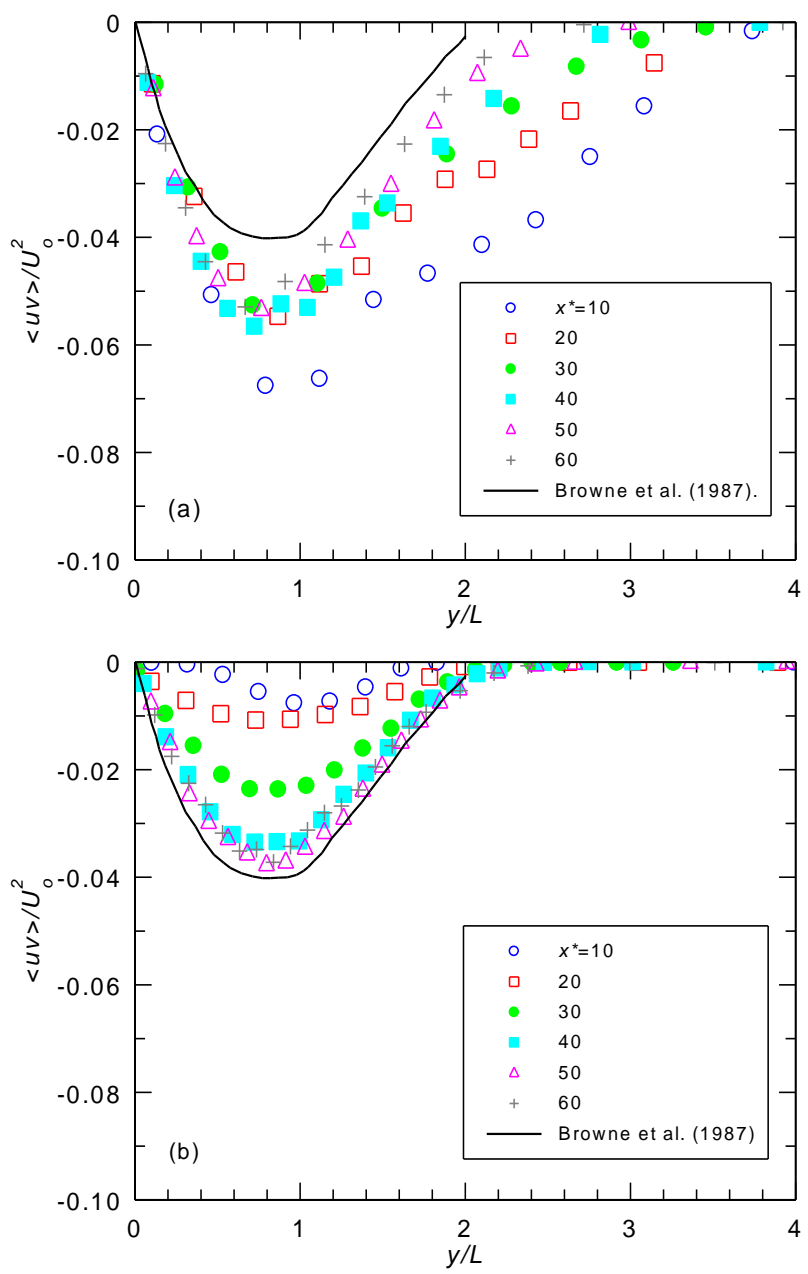

Fig. 5. Streamwise evolution of the normalized Reynolds shear stress. (a) solid cylinder; (b) screen cylinder. The solid lines represent values of the solid cylinder far wake at $x^{*}=420$ where self-preservation is achieved (Browne et al., 1987). 

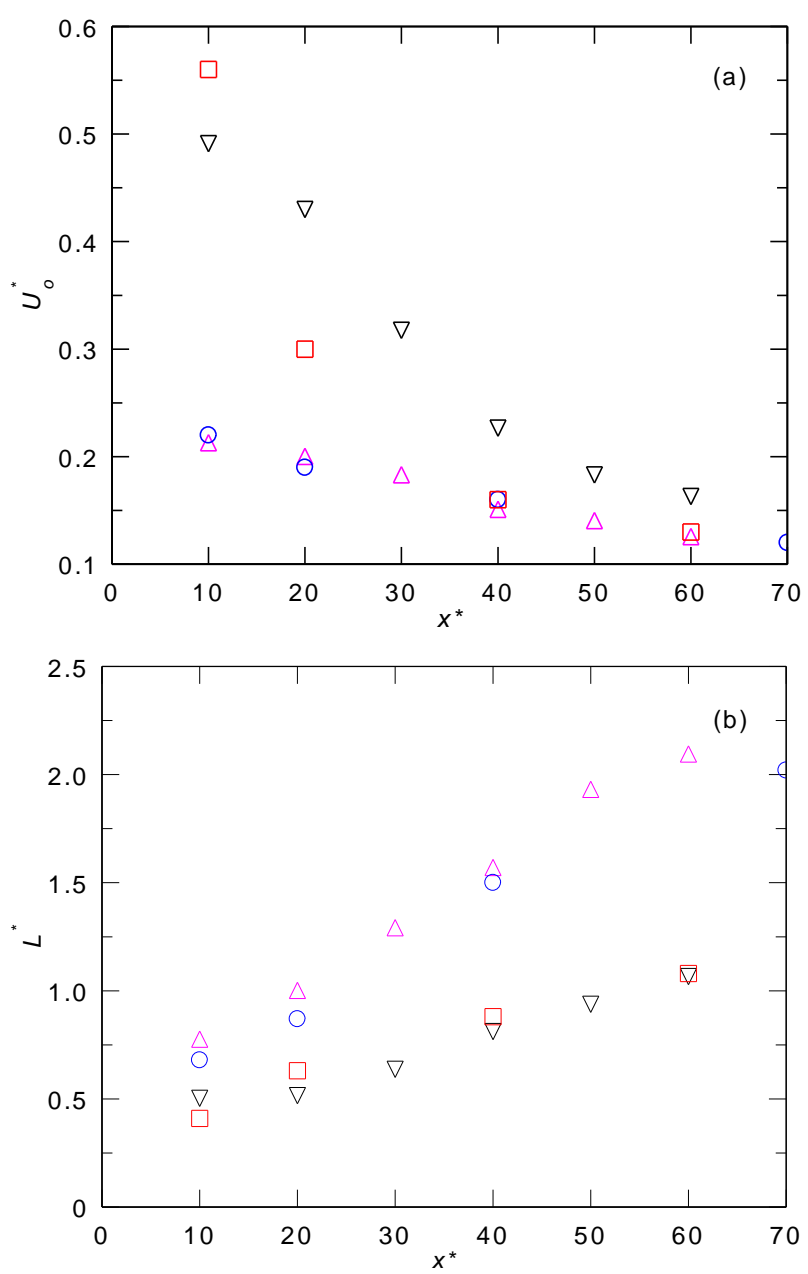

Fig. 6. Streamwise evolution of the velocity deficit and wake half-width. (a) velocity deficit; (b) wake half-width. $\Delta$, present solid cylinder $(R e=7000$ ); O, solid cylinder (Antonia and Mi, 1998); $\nabla$, present screen cylinder $(67 \%$ porosity and $R e=7000)$; $\square$ : screen strip (Antonia and $\mathrm{Mi}, 1998,50 \%$ porosity, $R e=3000$ ).

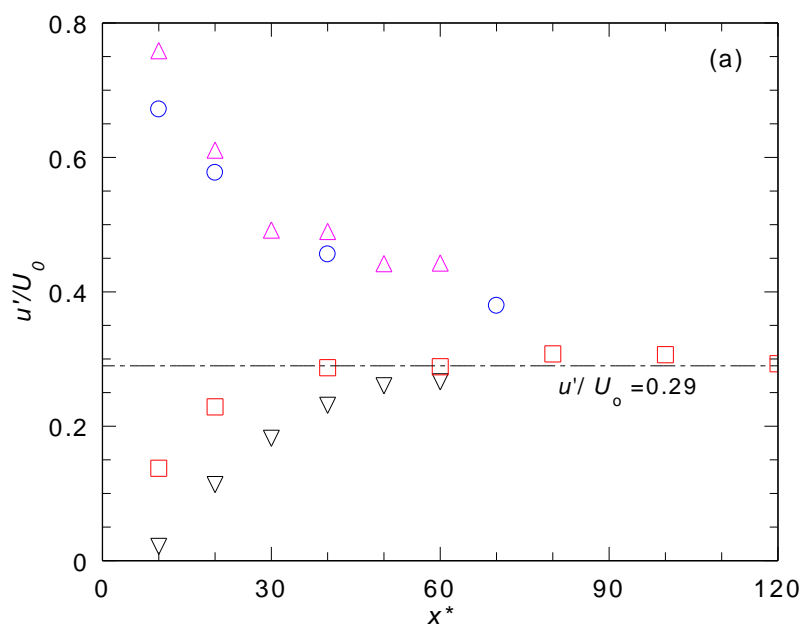




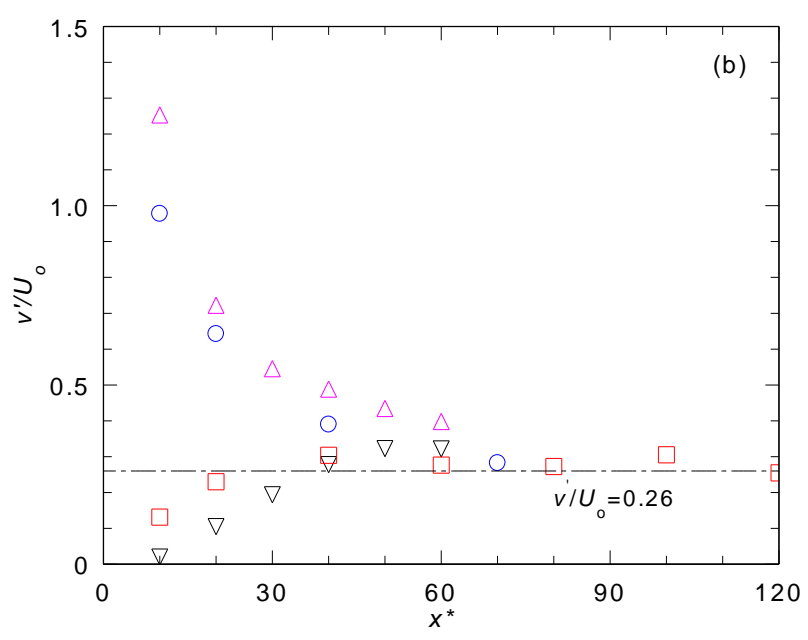

Fig. 7. Comparison of streamwise variation of $u^{\prime} / U_{o}$ and $v^{\prime} / U_{o}$ on the centreline of different wakes. (a) $u^{\prime} / U_{o}$; (b) $v^{\prime} / U_{o} . \Delta$, present solid cylinder $(R e=7000)$; O, solid cylinder (Antonia and $\mathrm{Mi}, 1998) ; \nabla$, present screen cylinder (67\% porosity and $R e=7000)$; $\square$ : screen strip (Antonia and $\mathrm{Mi}, 1998,50 \%$ porosity, $R e=3000$ ). The horizontal dash-dotted lines represent values in the solid cylinder far wake at $\operatorname{Re}=1170$ for $x^{*}=420$ where self-preservation is achieved (Browne et al., 1987).
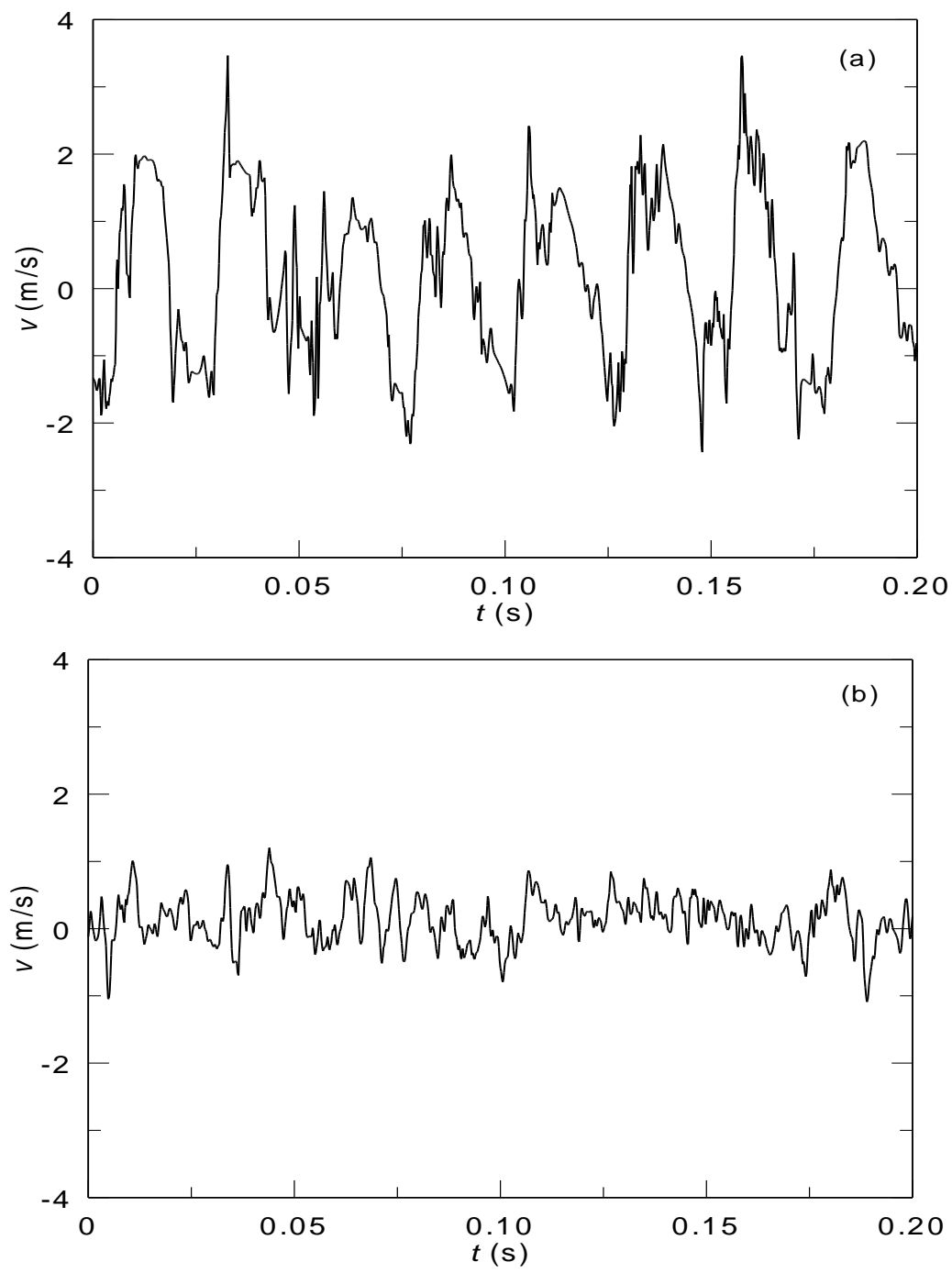


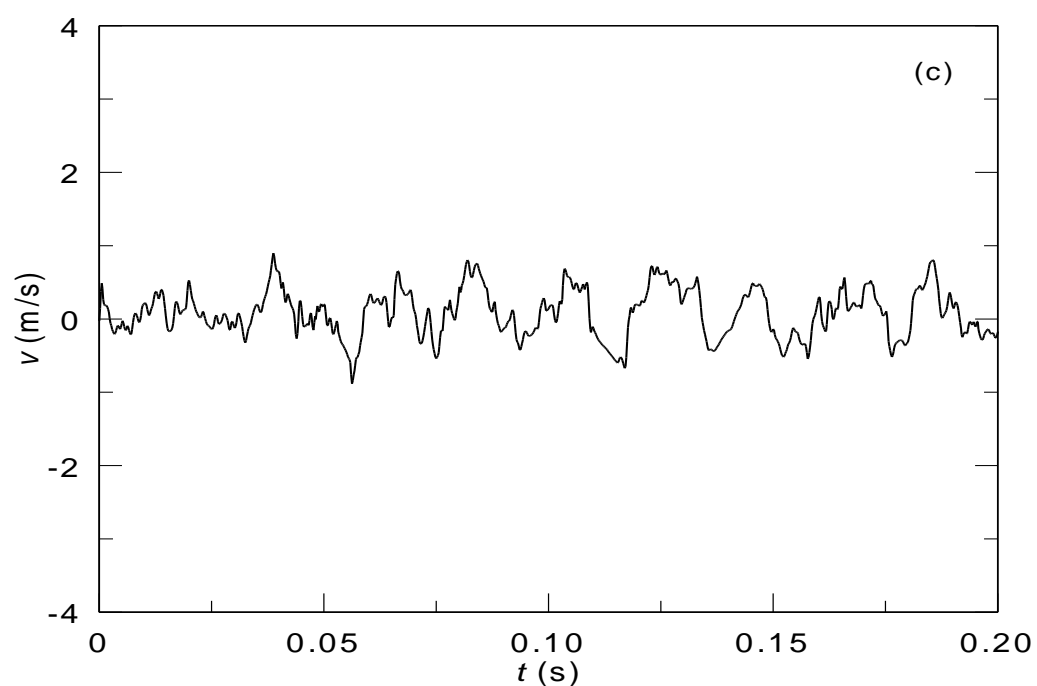

Fig. 8. Velocity signals obtained at $y^{*}=0.5$ for (a) solid cylinder wake at $x^{*}=10$; (b) screen cylinder wake at $x^{*}=10$ and (c) screen cylinder wake at $x^{*}=40$.
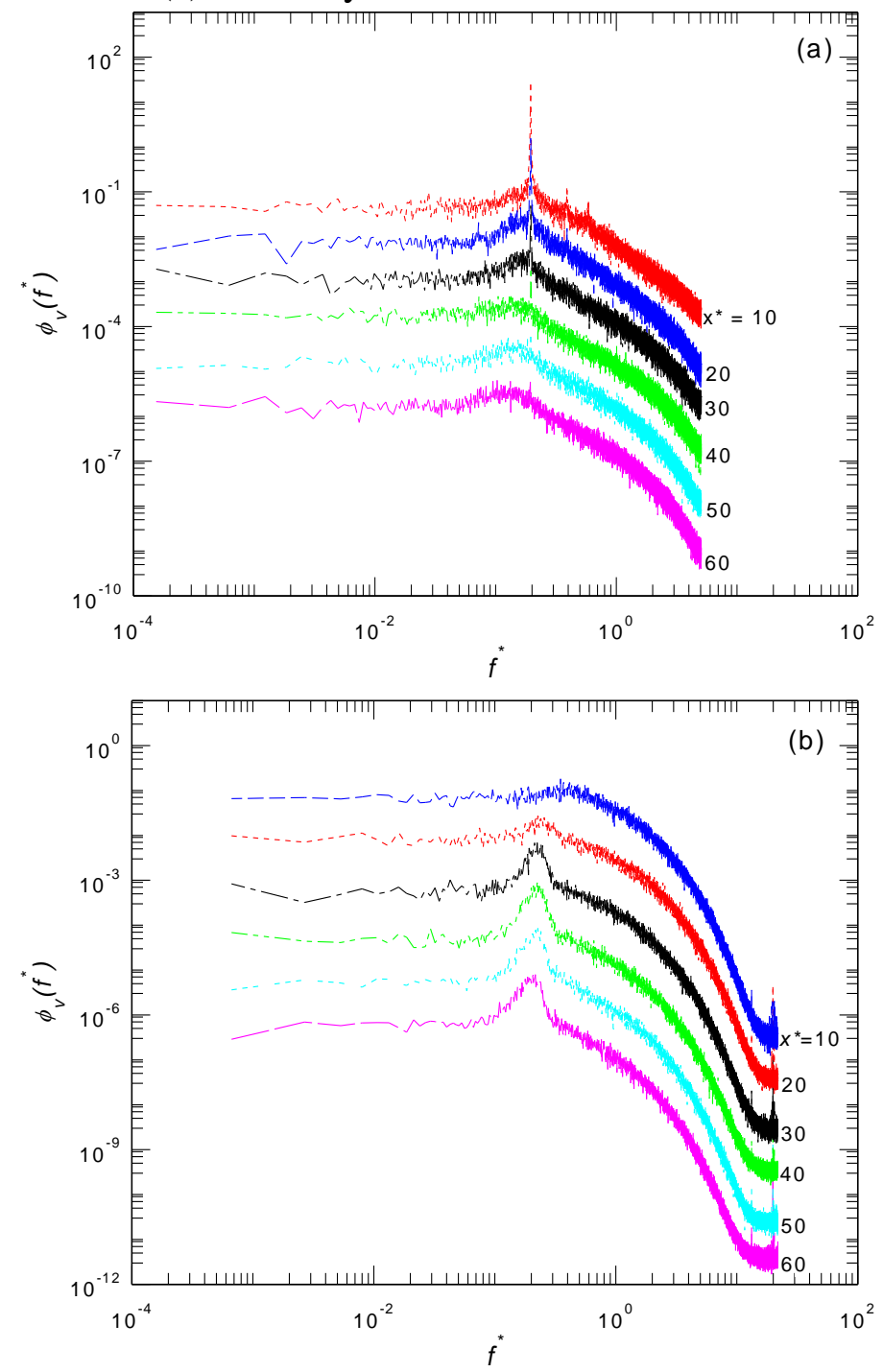


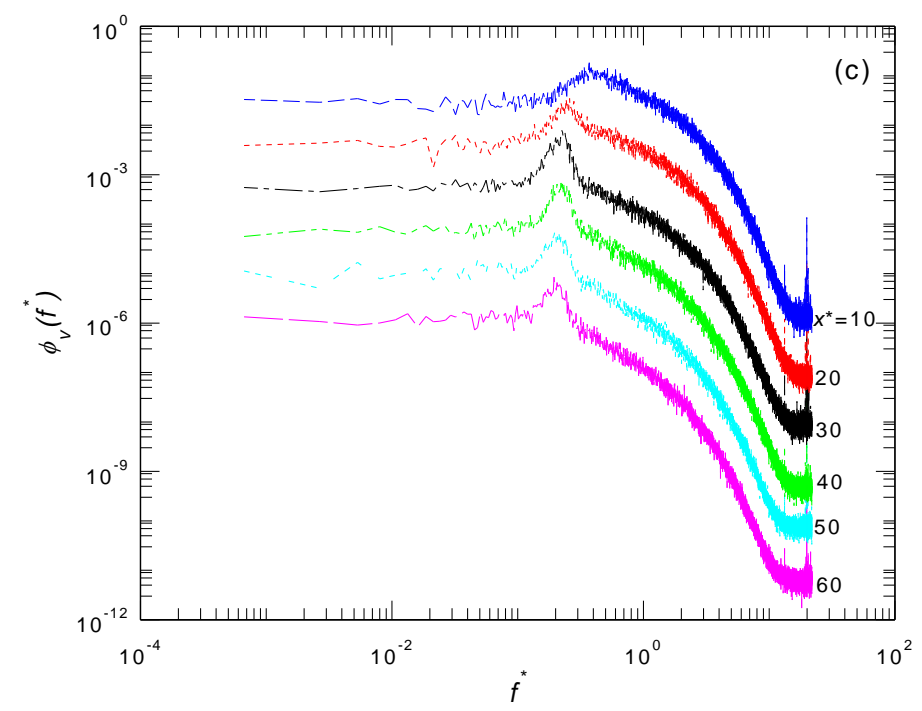

Fig. 9. Energy spectra $\phi_{v}$ in the wake of (a) solid cylinder measured at $y^{*}=0.5$; (b) screen cylinder measured at $y^{*}=0.5$ and (c) screen cylinder measured at the wake edge.

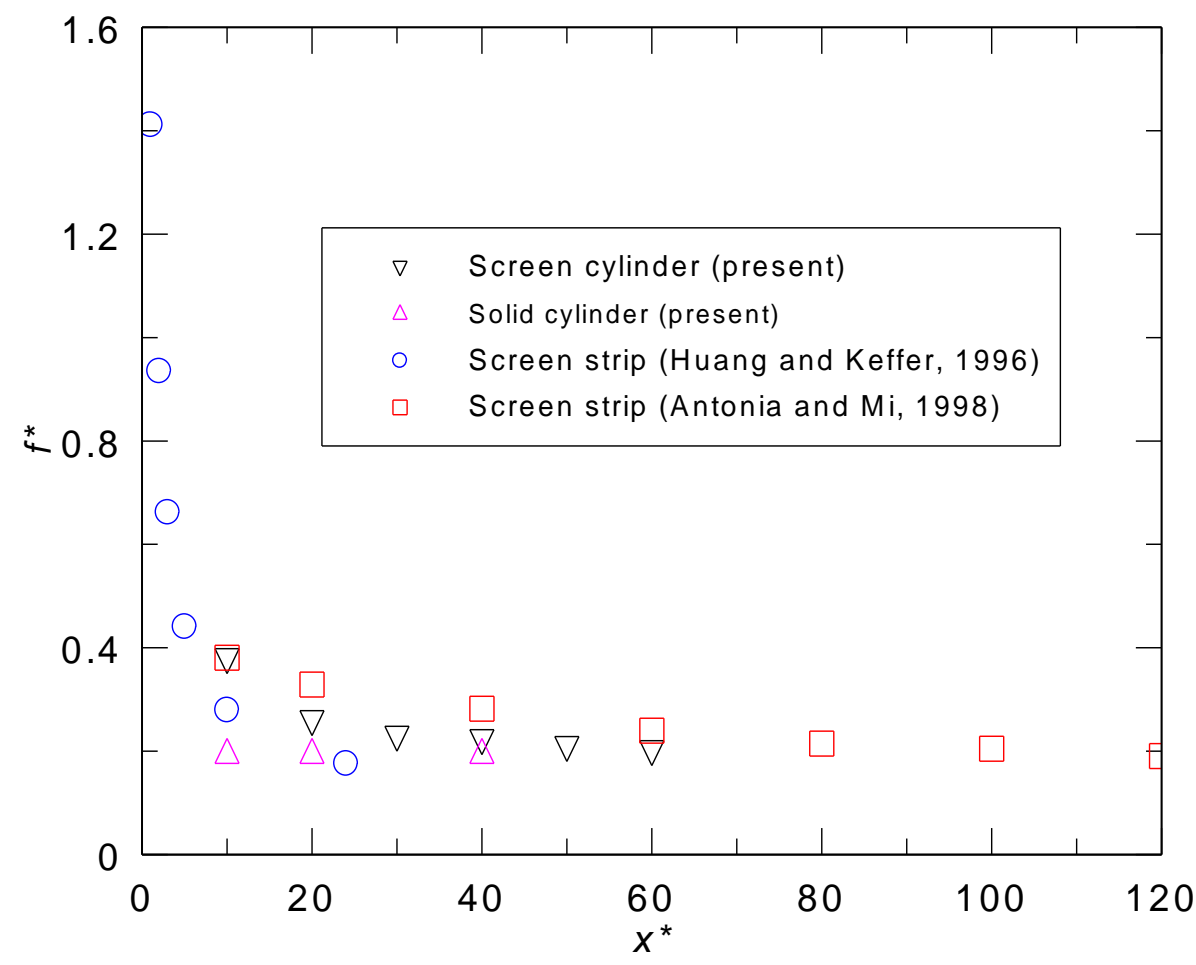

Fig. 10. Comparison of streamwise variation of the peak frequency in the solid cylinder, screen cylinder and screen strip wakes. $\nabla$, present screen cylinder $(67 \%$ porosity and $R e=$ $7000) ; \Delta$, present solid cylinder $(R e=7000) ;$ O, screen strip (Huang and Keffer, 1996) (40\% porosity and $R e=11,000$ ); $\square$ : screen strip (Antonia and Mi, 1998) (50\% porosity and $\operatorname{Re}=3000)$. 

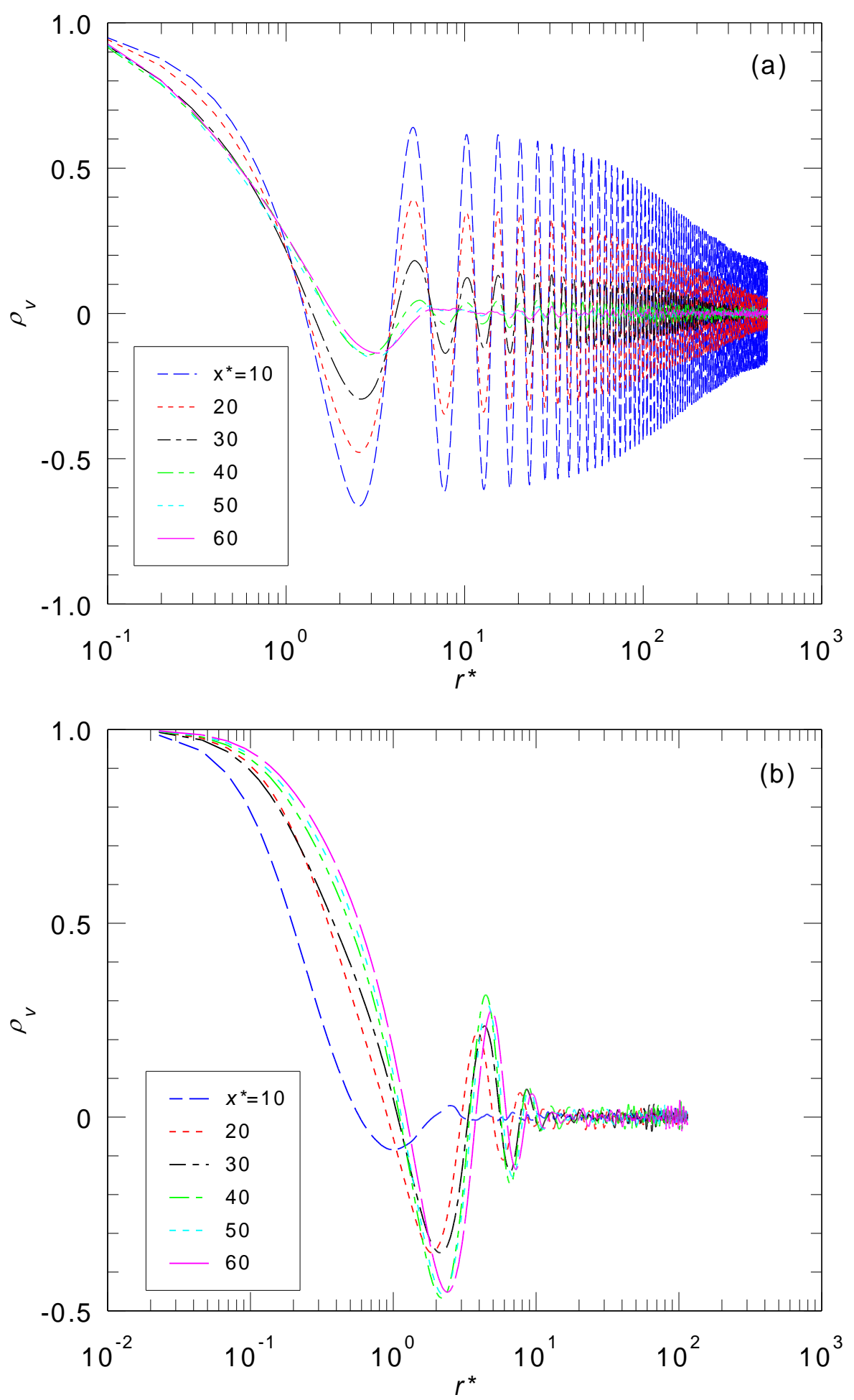

Fig. 11. Autocorrelation coefficient $\rho_{v}$ measured at $y^{*}=0.5$ at different downstream locations. (a) solid cylinder wake; (b) screen cylinder wake. 

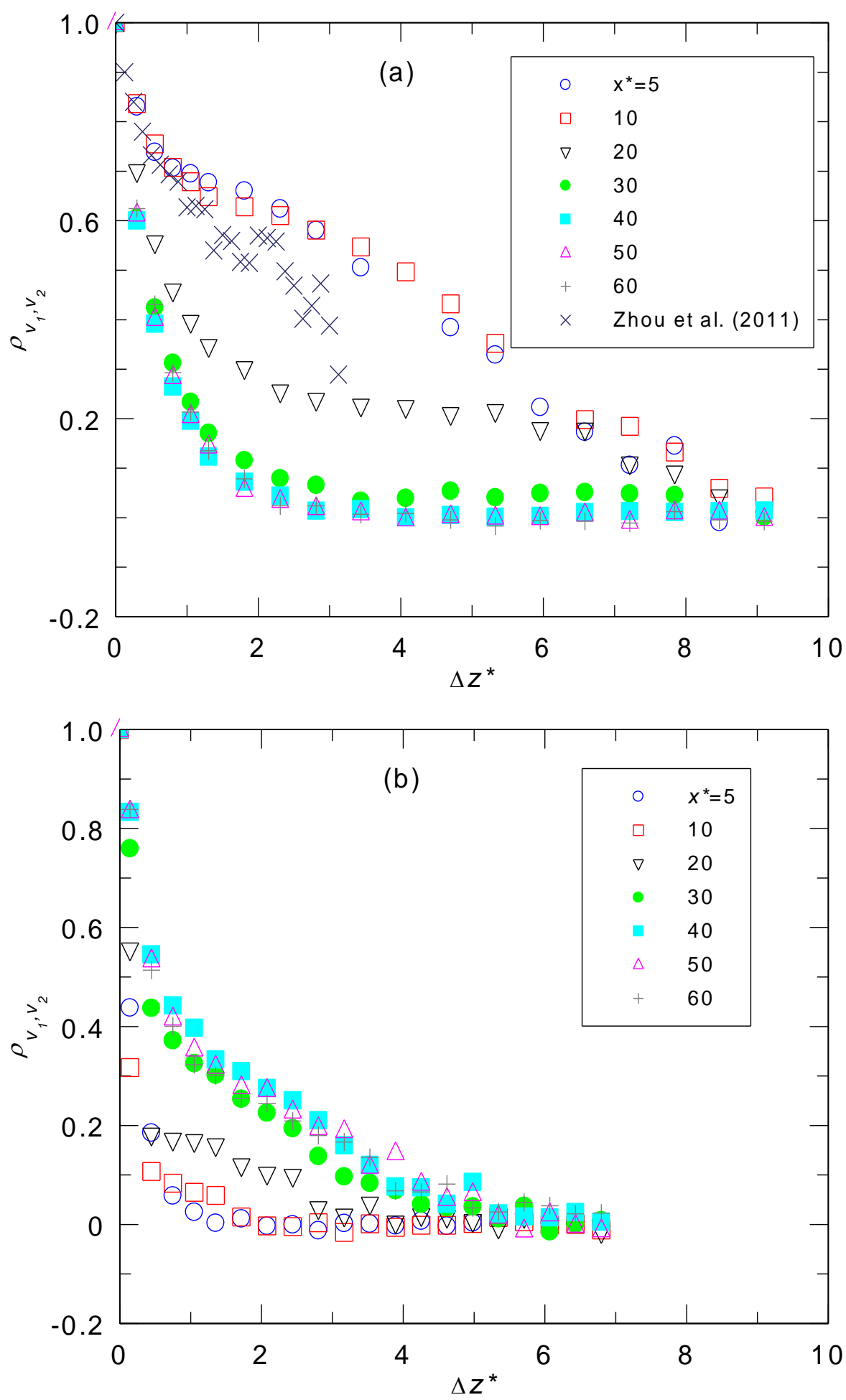

Fig. 12. Cross-correlation coefficient at different downstream locations. (a) solid cylinder wake; (b) screen cylinder wake. Experimental data of Zhou et al. (2011) were obtained at $x^{*}=$ 5 and $\operatorname{Re}=21,430$. 


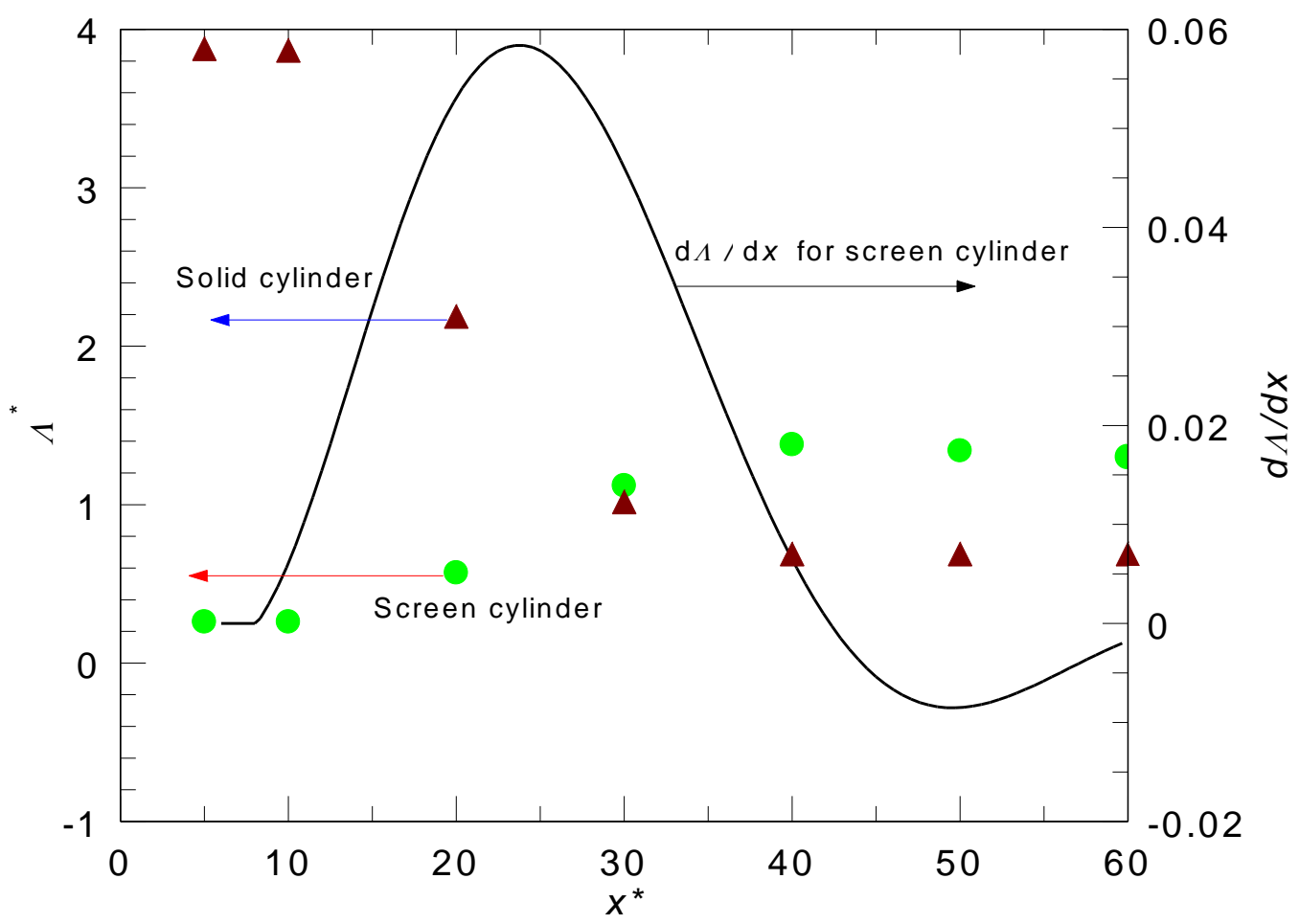

Fig. 13. Streamwise variation of the correlation length $\Lambda$ in the wakes of the solid cylinder and the screen cylinder and the gradient of $\Lambda$ in the screen cylinder wake. 

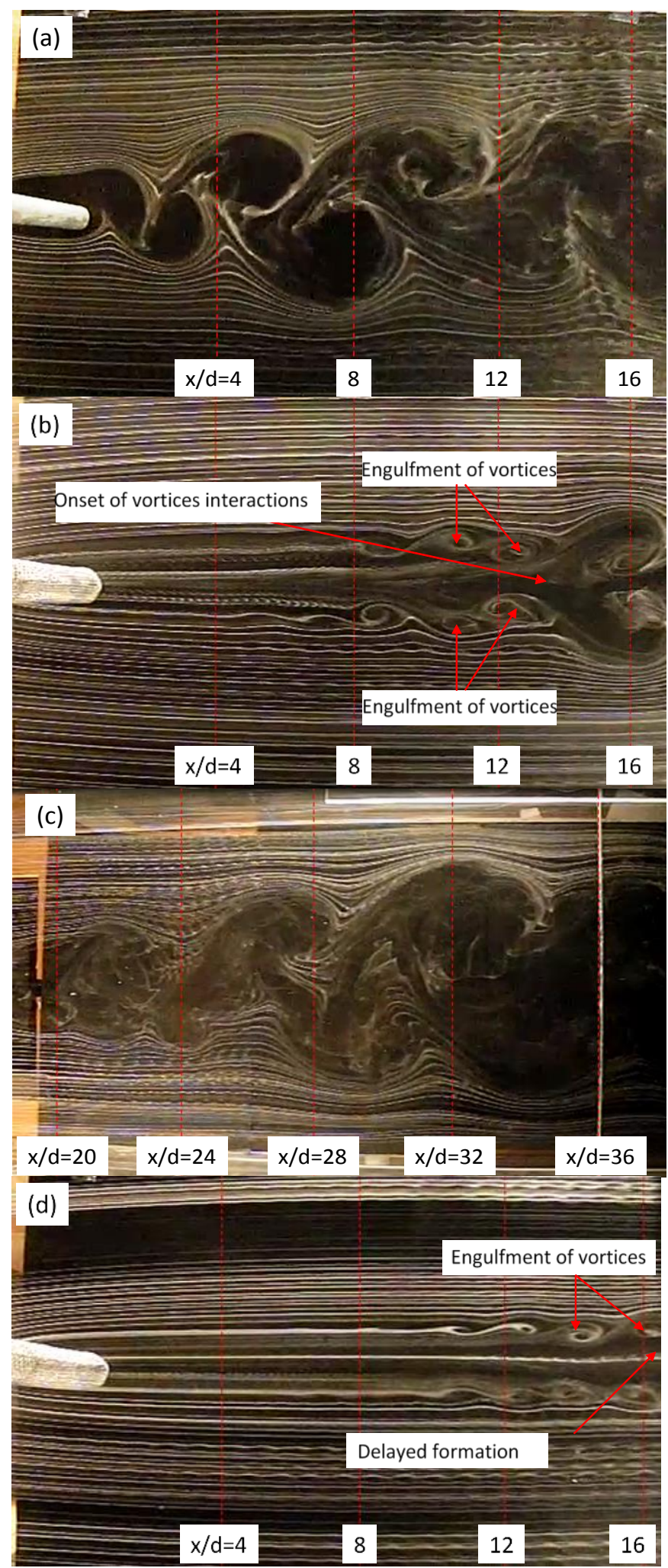

Figure 14. Flow visualization in the wakes of (a) solid cylinder $(R e=1240)$; (b) screen cylinder $(R e=1240)\left(x^{*}=0-16\right)$; (c) screen cylinder $(1240)\left(x^{*}=20-36\right)$ and (d) screen cylinder $(\operatorname{Re}=1920)$. 
